Journal for

... ImmunoTherapy of Cancer

\section{Mesothelin-specific CAR-T cell therapy that incorporates an HLA-gated safety mechanism selectively kills tumor cells}

To cite: Tokatlian T, Asuelime GE, Mock J-Y, et al. Mesothelin-specific CAR-T cell therapy that incorporates an HLA-gated safety mechanism selectively kills tumor cells. Journal for ImmunoTherapy of Cancer 2022;10:e003826. doi:10.1136/jitc-2021-003826

- Additional supplemental material is published online only. To view, please visit the journal online (http://dx.doi.org/10. 1136/jitc-2021-003826).

Accepted 20 December 2021
Check for updates

(C) Author(s) (or their employer(s)) 2021. Re-use permitted under CC BY-NC. No commercial re-use. See rights and permissions. Published by BMJ.

A2 Biotherapeutics Inc, Agoura Hills, California, USA

Correspondence to

Dr Alexander Kamb;

akamb@a2biotherapeutics.com

\section{ABSTRACT}

Background Mesothelin (MSLN) is a classic tumorassociated antigen that is expressed in lung cancer and many other solid tumors. However, MSLN is also expressed in normal mesothelium which creates a significant risk of serious inflammation for MSLN-directed therapeutics. We have developed a dual-receptor (Tmod $\left.{ }^{\mathrm{TM}}\right)$ system that exploits the difference between tumor and normal tissue in a subset of patients with defined heterozygous gene loss $(\mathrm{LOH})$ in their tumors.

Methods T cells engineered with the MSLN CAR Tmod construct described here contain (1) a novel MSLNactivated CAR and (2) an HLA-A*02-gated inhibitory receptor (blocker). $A^{*} 02$ binding is intended to override T-cell cytotoxicity, even in the presence of MSLN. The Tmod system is designed to treat heterozygous HLA class I patients, selected for HLA LOH. When $\mathrm{A}^{*} 02$ is absent from tumors selected for $\mathrm{LOH}$, the MSLN Tmod cells are predicted to mediate potent killing of the MSLN(+)A*02(-) malignant cells.

Results The sensitivity of the MSLN Tmod cells is comparable with a benchmark MSLN CAR-T that was active but toxic in the clinic. Unlike MSLN CAR-T cells, the Tmod system robustly protects surrogate "normal" cells even in mixed-cell populations in vitro and in a xenograft model. The MSLN CAR can also be paired with other HLA class I blockers, supporting extension of the approach to patients beyond $A^{\star} 02$ heterozygotes.

Conclusions The Tmod mechanism exemplified by the MSLN CAR Tmod construct provides an alternative route to leverage solid-tumor antigens such as MSLN in safer, more effective ways than previously possible.

\section{INTRODUCTION}

Immune cells are uniquely suited to confront the major impediment to better solid-tumor treatments: the shortage of tumor-specific molecular targets. A notable opportunity involves targeting multiple antigens to trigger more selective T-cell responses (reviewed in Dannenfelser et $a l^{1}$ ). Because gene lossespecially heterozygous gene loss $(\mathrm{LOH})$ - is common in solid tumors (figure 1A), it may be possible to exploit such irreversible genetic differences between tumor and normal cells with a dual-signal integrator. ${ }^{2}$ Indeed, a twopart mechanism to detect "missing self" based on families of activating and inhibitory receptors evolved in the early vertebrate immune system as a defense against pathogens (see for review Valiante $e t a \hat{l}$ ). Appropriate design and optimization of such a system could mitigate the safety issues of therapeutics targeting antigens that are expressed in tumors but constrained by expression on key normal tissues. $^{4-7}$

Mesothelin (MSLN) was proposed as a cancer target in 1992, ${ }^{8}$ yet there is still no viable therapy that uses MSLN. It is expressed on most mesotheliomas and also large subsets of ovarian, cervical, uterine, gastric, pancreatic, and lung adenocarcinomas. ${ }^{9}$ In normal adults, MSLN is present only in mesothelium, a tissue that itself may be nonessential. ${ }^{9} 10$ However, immunotherapy targeting MSLN carries the risk of inflammatory attack on MSLN (+) mesothelial cells that surround important internal organs. ${ }^{11}$ Several investigational therapeutics directed at MSLN have been tested; for example, immunotoxin conjugates, ${ }^{12} 13$ antibody-drug conjugates $^{14}$ (https://clinicaltrials.gov/ct2/show/ NCT01439152), bispecific antibodies, ${ }^{15}$ CARTs, ${ }^{16}$ and a hybrid TCR-scFv. ${ }^{17}$ Active systemically administered MSLN CAR-Ts display toxicity, and drug developers are struggling to find a solution. ${ }^{16} 18$ In a recent study, a MSLN CAR-T based on the M5 scFv caused respiratory failure at the highest dose (Tanyi et al, Penn Medicine presentation, 2021; https:/ / clinicaltrials.gov/ct2/show/NCT03054298). In another report, a MSLN-targeted chimeric scFv-TCR-T displayed mixed activity, but dosing was limited by toxicity, including cytokine release syndrome (Gavo-cel Phase 1/2 Clinical Update, TCR2.com; https:// 
clinicaltrials.gov/ct2/show/NCT03907852). Recently, an approach to deliver MSLN CAR-Ts via intrapleural infusion has been reported. ${ }^{19}$

We have developed a method to treat MSLN $(+)$ cancers that does not depend on local administration or tuneddown sensitivity, but instead exploits LOH. This approach seeks to avoid systemic toxicity to normal tissues by pairing a MSLN-targeted CAR with a LIR-1-based inhibitory receptor that blocks cytotoxicity in normal cells that express the $\mathrm{A}^{*} 02$ allele (figure $1 \mathrm{~A}, \mathrm{~B}$ ). This dual-receptor construct is intended to treat patients with genetically defined cancer who have clonal $\mathrm{LOH}$ of the $\mathrm{A} * 02$ allele in their MSLN (+) tumor. The difference in $\mathrm{A}^{*} 02$ expression in tumor versus normal tissues caused by $\mathrm{LOH}$ creates an all-or-nothing input to the effector cells that confers high selectivity for tumor killing.

\section{METHODS}

\section{Cell line culture}

Cell lines were purchased from ATCC (American Type Culture Collection) and handled according to manufacturer's instructions: T2, Shp77, Raji, MS751, A-375, A-498, SW982, SW480, HeLa, NIH-OVCAR-3, HepG2, NCI-H508, LNCaP clone FGC, K562, U2OS, BB7.1, and GAP A3 hybridomas. Jurkat cells encoding an NFAT luciferase reporter gene were obtained from BPS Bioscience and maintained in RPMI supplemented with $10 \%$ heat-inactivated (HIA) FBS (inactivated at $56^{\circ} \mathrm{C}$ for 1 hour), $1 \%$ penicillin and streptomycin (pen/strep), and $0.4 \mathrm{mg} / \mathrm{mL}$ Geneticin. Human peripheral blood mononuclear cells (PBMCs) were obtained from Stem Cell Technologies and thawed in X-VIVO 15 (Lonza) supplemented with 5\% HIA human $\mathrm{AB}$ serum and activated with T Cell TransAct (Miltenyi Biotec) as recommended by the manufacturer. Lentiviral transductions of CAR or CAR Tmod single vector constructs (see figure 1B, unless otherwise noted) were done at 24 hours post-activation and cultures were maintained in LymphoONE $\mathrm{T}$ cell Expansion Medium (Takara Bio) supplemented with 1\% HIA human AB serum and 300 IU/mL IL-2.

\section{Molecular cloning and mRNA in vitro transcription}

Activating and blocking CAR constructs were designed and constructed as previously described. ${ }^{6}$ Briefly, activating CARs were created by fusing the anti-MSLN scFv LBDs to the CD8 $\alpha$ hinge, CD28 transmembrane (TM), and $\mathrm{CD} 28,4-1 \mathrm{BB}$, and $\mathrm{CD} 3 \zeta$ intracellular domains (ICD). Blocking CARs were generated by fusing the anti-HLA-A $* 02, A * 03$, or $B * 07$ scFv LBDs derived from monoclonal antibodies PA2.1, ${ }^{20}$ GAP A3, ${ }^{21}$ or BB7.1, ${ }^{22}$ respectively, to the hinge, TM, and ICD domains of LIR-1. A humanized PA2.1 blocker was generated by complementarity determining region (CDR) grafting onto a human framework. Gene segments were combined using Golden Gate cloning and inserted downstream of a human EF1 $\alpha$ promoter in a lentiviral expression vector. To downregulate B2M, an expression construct was generated with a
B2M-targeting shRNA driven from the U6 promoter and placed upstream of the EF1 $\alpha$ promoter.

For messenger RNA (mRNA) preparation, PCR was used to generate the DNA template for in vitro synthesis mRNA. Briefly, T7 promoter was introduced via N-terminal primer and common overhang regions were used for both N-terminal and C-terminal primers. The PCR product was used as a template for in vitro transcription (IVT) using the T7 ARCA mRNA kit (New England Biolabs, E2060S). The IVT reaction comprised 1× ARCA/ NTP mix, 1.25 mM $\Psi$-UTP (TriLink), $25 \mu \mathrm{g}$ PCR product template, and $1 \times$ T7 RNA polymerase. The IVT reaction was incubated at $37^{\circ} \mathrm{C}$ for 2 hours, then $2 \mu \mathrm{L}$ of DNase I (New England Biolabs, M0303S) was added to each reaction and incubated at $37^{\circ} \mathrm{C}$ for $15 \mathrm{~min}$. To the IVT reaction, $65 \mu \mathrm{L}$ water, $10 \mu \mathrm{L} 10 \times$ poly-adenylation reaction buffer (NEB M0276S), and $5 \mu \mathrm{L}$ of poly-adenylation enzyme (NEB M0276S) were added to a total volume of $\sim 100 \mu \mathrm{L}$. The reaction was incubated at $37^{\circ} \mathrm{C}$ for $30 \mathrm{~min}$. The resulting product was cleaned using the Monarch RNA cleanup kit (T2040L) according to the manufacturer's protocol.

\section{MSLN and HLA-A ${ }^{\star} 11$ binder generation using mammalian display}

Binder generation using the HuTARG ${ }^{\mathrm{TM}}$ mammalian display technology has been previously described (Patent No: US 8,012,714 B2) ${ }^{23}$ For the MSLN binder generation, soluble MSLN (catalog \#MSN-H82E9) and irrelevant, off-target proteins, EGFR and CEA, were purchased from Acro Biosystems. The HuTARG library was subjected to serial rounds of on-target enrichment and off-target depletion using fluorescence-activated cell sorting (FACS). The A*11 binder generation campaign was similarly conducted. The HuTARG library was subjected to on-target enrichment using $A^{*} 11: 01$ tetramer and offtarget depletion using a pool of four unrelated HLA class I tetramers. In the final round of both campaigns, on-target and off-target binding cells were collected, and RNA was reverse transcribed into cDNA. Fragments containing the CDRs were amplified using PCR and sequenced by nextgeneration sequencing (NGS). Binders of interest were selected by comparing the input and output NGS counts.

\section{MSLN surface quantification}

To quantify surface mesothelin molecules, adherent cells were washed with DPBS,coated with Versene and incubated at $37^{\circ} \mathrm{C}$ for $\sim 15 \mathrm{~min}$. After incubation, flasks were tapped vigorously to promote complete detachment. The cells were diluted with DBPS at 1:2 ratio (Versene:DPBS) and counted. A total of 300,000 cells were centrifuged at $500 \times g$ for $5 \mathrm{~min}$. Cells were resuspended in $300 \mu \mathrm{L} 1 \times$ FACS buffer (DPBS $+1 \%$ BSA). One hundred microliters of aliquot was divided into two v-bottom wells. Cells were washed one more time with $100 \mu \mathrm{L}$ FACS buffer, then stained with $100 \mu \mathrm{L}$ of anti-MSLN antibody at $10 \mu \mathrm{g} / \mathrm{mL}$ (R\&D Systems, clone 618923) on ice for $30 \mathrm{~min}$. After the primary stain, $50 \mu \mathrm{L}$ of calibration beads from the 
A

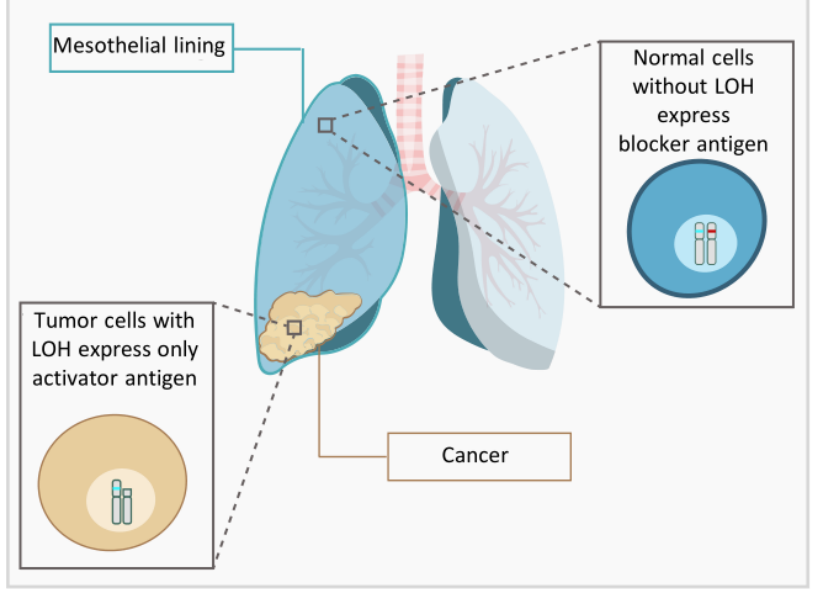

C

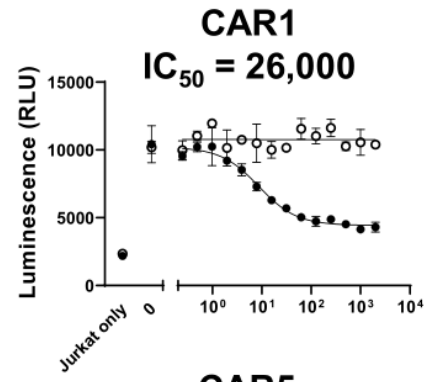

CAR5
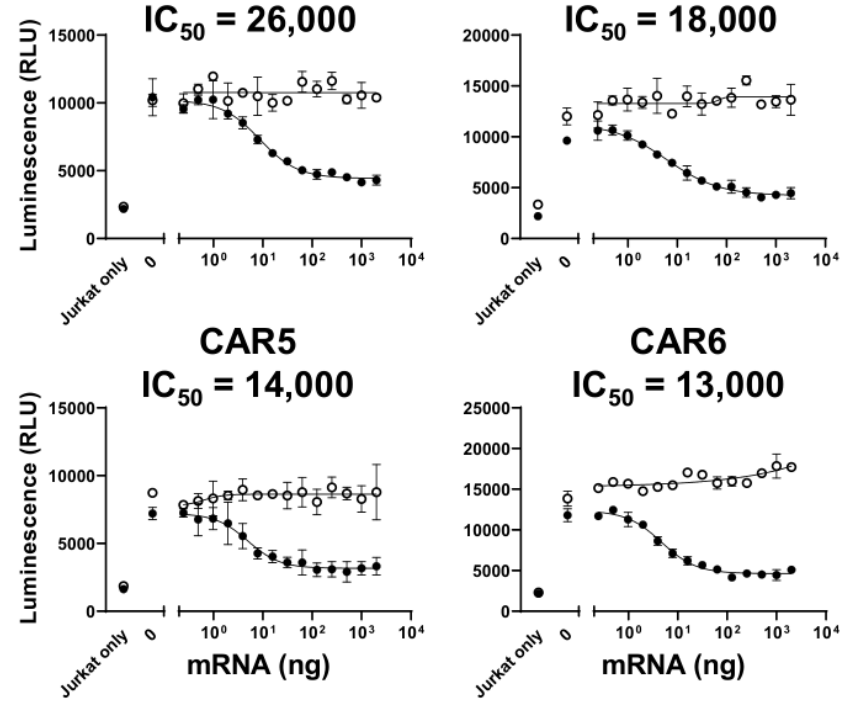

CAR6

$I_{50}=13,000$
B

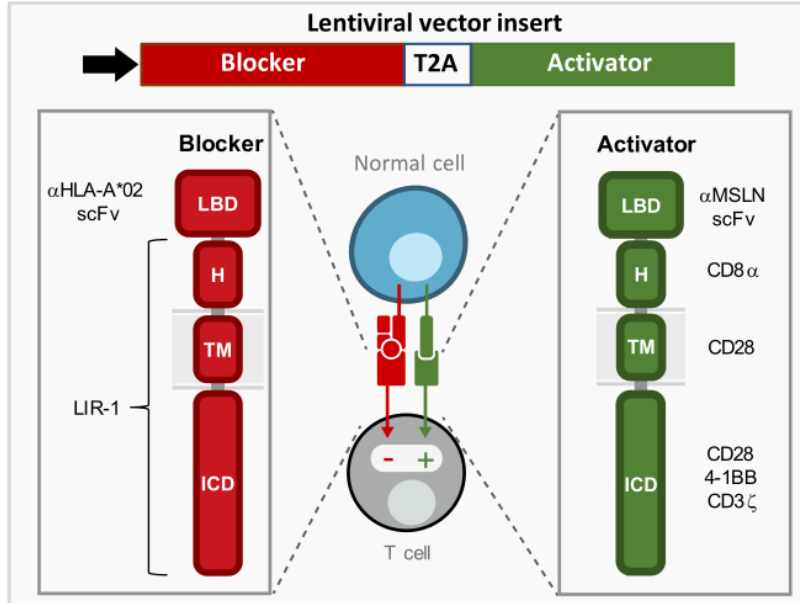

O CAR @ CAR TmOd

CAR3

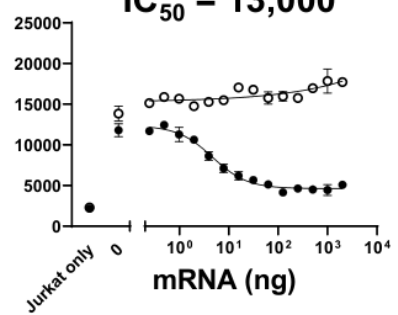

$I C_{50}=\mathbf{2 9 , 0 0 0}$

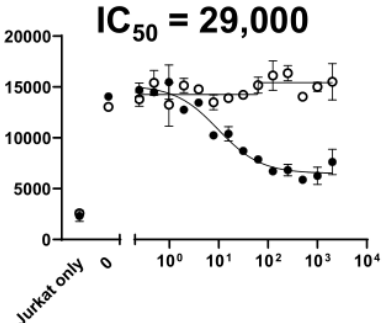

M5

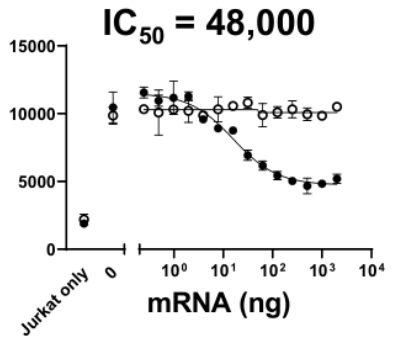

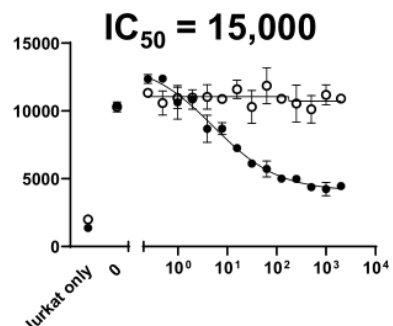

SS1

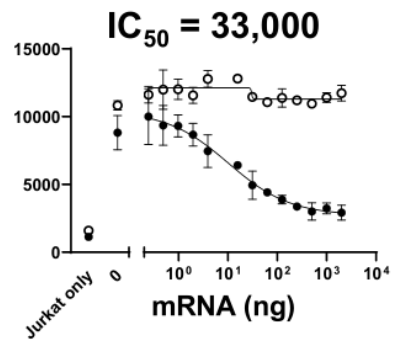

D
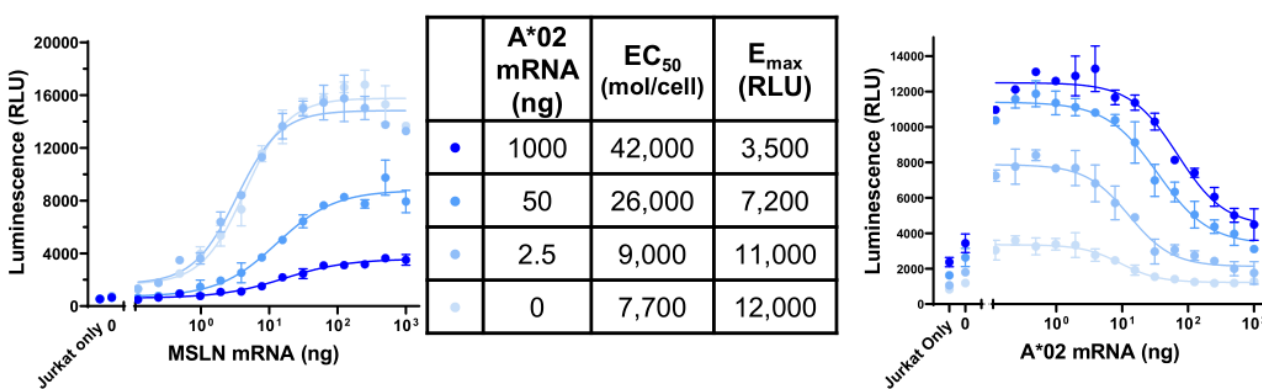

Figure 1 Tmod approach to achieve selective cytotoxicity with two targets. (A) The lung (and other vital organs) are surrounded by the MSLN(+) mesothelial lining, creating high risk of on-target, off-tumor toxicity for MSLN-targeted medicines. Selecting patients heterozygous for $\mathrm{A}^{*} 02$ whose tumors have lost this allele via LOH gives an opportunity to target MSLN selectively with CAR Tmod cells so they kill tumor cells specifically and spare normal mesothelium. (B) Molecular composition of MSLN-targeted Tmod constructs. The two receptors are co-expressed in a single construct and the encoded fusion protein is cleaved in the cell to generate the activator and blocker. (C) Six display-derived MSLN activators (CAR1-6) and benchmark CARs M5 and SS1 activators were paired with $A^{*} 02$ blocker (solid) or empty vector control (open). Jurkat cells expressing the $\mathrm{CAR} \pm$ blocker (for receptor staining see online supplemental figure 1F) were co-cultured with endogenous MSLN(+) HeLa cells transfected with a titration of $A^{\star} 02: 01$ mRNA. Response was assessed after 6-hour co-culture. Titrated antigen molecules on the surface of target cells were quantified using the QIFIKIT. IC ${ }_{50}$ (molecules/cell) values are indicated in the figure. M5 and SS1 CARs are Gen2. (D) Two-dimensional titration of MSLN and $A^{*} 02$ mRNA in MSLN(-) HeLa target cells to establish EC and $_{50}$ and $\mathrm{IC}_{50}$ for the MSLN CAR3 Tmod construct in Jurkat cells. MSLN(-) HeLa target cells were transfected with serially diluted MSLN mRNA and constant $A^{*} 02$ mRNA (left) or serially diluted $A^{*} 02$ mRNA and constant MSLN mRNA (right). 
QIFIKIT (Agilent) were added. The mixture was washed with $100 \mu \mathrm{L}$ FACS buffer twice, then stained with $100 \mu \mathrm{L}$ of anti-mouse $\mathrm{F}\left(\mathrm{ab}^{\prime}\right)$ 2-Goat Alexa Fluor 647 (Invitrogen) antibody diluted $2000 \times$ in FACS buffer for $45 \mathrm{~min}$ on ice. The stained cells and beads were washed $2 \times$ with $100 \mu \mathrm{L}$ FACS buffer, then resuspended in $100 \mu \mathrm{L}$ FACS buffer to measure fluorescence. The calibration curve plotting median fluorescence intensity of each bead population versus number of molecules was plotted according to the manufacturer's protocol provided by the QIFIKIT (Agilent). The number of endogenous MSLN molecules was determined using the cell's MFI and the calibration curve generated with the QIFIKIT beads.

\section{MSLN activator and blocker sensitivity determination}

To determine activator or blocker sensitivities, antigen was titrated into antigen $(-)$ cell lines at various concentrations. Flow cytometry and QIFIKIT methodology ${ }^{24}$ using standard curves could then be used to calculate absolute molecules/ cell. The $\mathrm{EC}_{50}$ or $\mathrm{IC}_{50}$ value of activators or blockers, respectively, expressed in Jurkat cells was then measured by luminescence. Specifically, HEK293T or HeLa cell transfection with antigen mRNA was performed using the 4D nucleofection kit (Lonza). For single-antigen titrations (ie, MSLN, $\left.\mathrm{A}^{*} 02, \mathrm{~A}^{*} 11, \mathrm{~A}^{*} 03, \mathrm{~B}^{*} 07\right)$, mRNA was diluted to $1000 \mathrm{ng} /$ $\mu \mathrm{L}$ in SE or SF buffer supplemented with Supplement solution (complete buffer) for HEK293T or HeLa cells, respectively. The diluted mRNA was serially diluted $2 \times$ by adding stock mRNA to equal volumes of complete buffer 13 times, with the 15th well lacking any added mRNA. Target cells were detached from the flask using TrypLE Express (Gibco). Appropriate numbers of cells were harvested then resuspended in complete buffer at $11.1 \mathrm{e} 6$ viable cells $/ \mathrm{mL}$. Then $22.5 \mu \mathrm{L}$ of resuspended cells was added to $2.5 \mu \mathrm{L}$ of serially diluted mRNA to a final volume of $25 \mu \mathrm{L}$ cell and mRNA mix. Twenty microliters of this sample was zapped using the 4D nucleofector using the CM-130 program for HEK293T cells or CN-114 program for HeLa cells. The cells were transferred into $280 \mu \mathrm{L}$ of MEM $+10 \% \mathrm{FBS}+0.1 \% \mathrm{P} / \mathrm{S}$. Then $15 \mu \mathrm{L}$ of the diluted cells were transferred into a $384-$ well plate and incubated $18-24$ hours at $37^{\circ} \mathrm{C}, 5 \% \mathrm{CO}_{2}$. The remaining diluted cells were transferred to a 96 -well plate and kept for next-day analysis of surface antigen expression using the QIFIKIT as described previously. In parallel, Jurkat-NFAT luciferase effector cells were transfected with appropriate CAR DNA at $1 \mu \mathrm{g}$ per $1 \mathrm{e} 6$ cells or CAR Tmod DNA (single vector construct, unless otherwise noted) at $4 \mu \mathrm{g}$ per $1 \mathrm{e} 6$ cells using the Neon electroporation system using the following parameters: $1500 \mathrm{~V}, 10 \mathrm{~ms}$, and 3 pulses. Transfected cells were immediately transferred to prewarmed RPMI supplemented with $20 \%$ heat-inactivated (HIA) FBS and $0.1 \%$ pen/strep and incubated for 18-24 hours at $37^{\circ} \mathrm{C}, 5 \% \mathrm{CO}_{2}$. Next, Jurkat cells were counted and resuspended in RPMI $+10 \%$ HIA FBS and $0.1 \%$ pen/strep at $0.67 \mathrm{e} 6$ cells $/ \mathrm{mL}$. Resuspended Jurkat cells (1e4) in $15 \mu \mathrm{L}$ were then co-cultured with transfected cells in the 384-well plate for 6 hours. Luciferase activity was measured using ONE-Step Luciferase Assay System (BPS Bioscience).
For target cell co-transfections including MSLN and $\mathrm{A}^{*} 02$, the aforementioned protocol was used except for minor adjustments to the mRNA dilutions step. mRNA encoding $A^{*} 02$ and MSLN were prepared by Aldevron and TriLink, respectively. Briefly, to generate $\mathrm{EC}_{50}$ curves, a 14-point $2 \times$ serial dilution was performed with MSLN mRNA. This serial dilution was combined with each of four dilutions of various constant amounts of $\mathrm{A} * 02 \mathrm{mRNA}$ so that the top concentration of MSLN mRNA was $500 \mathrm{ng} / \mu \mathrm{L}$ and the $\mathrm{A}^{*} 02$ mRNA concentrations were as follows: 500 $\mathrm{ng} / \mu \mathrm{L}, 25 \mathrm{ng} / \mu \mathrm{l}, 1.25 \mathrm{ng} / \mu \mathrm{L}$, and $0 \mathrm{ng} / \mu \mathrm{L}$. To generate $\mathrm{IC}_{50}$ curves, a 14-point $2 \times$ serial dilution was performed with $A^{*} 02$ mRNA. This serial dilution was combined with each of four dilutions of various constant amounts of MSLN mRNA so that the top concentration of A*02 mRNA was $500 \mathrm{ng} /$ $\mu \mathrm{L}$ and the MSLN mRNA concentrations were as follows: $125 \mathrm{ng} / \mu \mathrm{L}, 25 \mathrm{ng} / \mu \mathrm{L}, 5 \mathrm{ng} / \mu \mathrm{L}$, and $1 \mathrm{ng} / \mu \mathrm{L}$.

\section{Primary $\mathrm{T}$ cell in vitro cytotoxicity assays}

Killing of MSLN $(+) \mathrm{A}^{*} 02(-) \mathrm{RFP}(+)$ tumor or MSLN $(+)$ $\mathrm{A}^{*} 02(+) \mathrm{GFP}(+)$ "normal" target cells by primary $\mathrm{T}$ cells transduced with Gen2 M5 CAR, MSLN CAR3, or CAR3 Tmod single vector constructs was assessed as previously described. ${ }^{6}$ Briefly, 2000 target cells were plated in $25 \mu \mathrm{L}$ complete LymphoOne media (containing 1\% HIA human $\mathrm{AB}$ serum) per well in a black, clear-bottom 384-well plate and allowed to adhere overnight at $37^{\circ} \mathrm{C}$ with $5 \% \mathrm{CO}_{2}$. For mixed culture assays, target cells were pre-mixed at the desired ratios prior to seeding. Approximately $16-18$ hours after target cell plating, $\mathrm{T}$ cells were counted, spun down at $300 \times g$ for $10 \mathrm{~min}$ and resuspended at $2000 \mathrm{CAR}(+)$ or Tmod(+) T cells in $25 \mu \mathrm{L}$ complete LymphoOne media (in the absence of additional cytokines) for an effective effector:target (E:T)=1:1 (or more or less concentrated depending on the desired E:T) and gently plated on top of the target cells. For experiments including sMSLN, T cells were resuspended in LymphoOne media containing $1000 \mathrm{ng} / \mathrm{mL}(2 \times)$ soluble, monomeric MSLN (Acro Biosystems) for a final concentration of $500 \mathrm{ng} / \mathrm{mL}$ on plating with target cells. Each sample was tested in triplicate wells. Within $30 \mathrm{~min}$ of co-culture, the plate was imaged for $\mathrm{GFP}(+)$ or $\mathrm{RFP}(+)$ target cell expression using an Incucyte imager and serial images were obtained every 2-4 hours thereafter for up to 48 hours. Quantification of target cell area (ie, GFP $(+)$ or $\mathrm{RFP}(+)$ total area per image) was done using Incucyte imaging software. Plating variability was accounted for by normalizing to area at time $=0$ per well. Killing was then quantified as the difference in area between CAR or Tmod $\mathrm{T}$ cell wells vs. corresponding untransduced $\mathrm{T}$ cell wells, normalized to the untransduced T cell well $\left(\%\right.$ Killing $\left.=\left(\mathrm{A}_{\text {untransduced }}-\mathrm{A}_{\text {CAR or Tmod }}\right) / \mathrm{A}_{\text {untransduced }}\right)$. For $A^{*} 11$ blocker evaluation in primary $\mathrm{T}$ cells, cytotoxicity assays were performed as described previously, using HeLa target cells that express native levels of MSLN and transgenic $A * 11$ antigen.

To further assess cytokine secretion and relative $\mathrm{T}$ cell activation post- $48 \mathrm{~h}$ co-culture, the $\mathrm{T}$ cell-containing media in each well was mixed and transferred to a 
v-bottom plate and spun down at $400 \times g$ for 5 min. Supernatants were collected and frozen until further analysis for secreted IFN- $\gamma$ using a BD Human IFN- $\gamma$ flex kit per manufacturer's instructions. Remaining T cells were then stained for human CD3, washed, and characterized for forward and side scatter by flow cytometry. ${ }^{25}$

\section{Repeat antigen-challenge assay (RACA) and reversibility assays}

The RACA and reversibility assays were performed as previously described with some modifications. ${ }^{6}{ }^{23}$ Briefly, CAR or Tmod transduced primary $\mathrm{T}$ cells were co-cultured with $\operatorname{MSLN}(+) \mathrm{A}^{*} 02(-) \quad \mathrm{RFP}(+)$ tumor or $\operatorname{MSLN}(+) \mathrm{A}^{*} 02(+)$ $\mathrm{GFP}(+)$ "normal" target cells similarly as described previously with effective E:T=1.2:1. Round 1 images were taken over the course of 48 hours in a 384-well plate format. Parallel 6-well plates with 250,000 target cells and 300,000 CAR3(+) or CAR3 Tmod(+) T cells were also set up to allow for bulk $\mathrm{T}$ cell isolation and transfer between rounds. After 48 hours, $\mathrm{T}$ cell-containing media was gently mixed and transferred to conical tubes. Remaining $\mathrm{T}$ cells engaged with live target cells were further dissociated by a single $1 \mathrm{~min}$ rinse with 10 $\mathrm{mM}$ EDTA $+0.5 \%$ BSA in PBS and combined with the bulk $\mathrm{T}$ cell fraction. These cells (along with target cell debris and lifted target cells) were then spun down, washed, and stained with a cocktail of anti-MSLN, anti-EGFR, and anti-N-cadherin PE-conjugated antibodies to stain undesirable target cells. These target cells were then conjugated to anti-PE MACS beads followed by depletion through LS columns, resulting in clean $\mathrm{T}$ cell fractions. T cells were counted and re-seeded for round 2 (similarly to round 1 ) onto fresh tumor or "normal" target cells. Imaging and quantification then proceeded exactly as was done in round 1 .

\section{MSLN knockout cell line generation}

To develop MSLN(-) cell line controls for endogenous MSLN (+) cell lines, a CRISPR strategy to target full-length MSLN was used. Two Alt-R CRISPR-Cas9 sgRNAs targeting different exons of mesothelin were obtained from Integrated DNA Technologies (IDT), with sgRNA_1 targeting exon 2, and sgRNA_2 targeting exon 16. The sgRNAs were rehydrated in nuclease-free water and combined with Alt-R S.p. HiFi Cas9 Nuclease V3 (IDT) to yield a 9:1 sgRNA:Cas9 mole ratio. Both RNP solutions were incubated at room temperature for 10-20 min separately to allow each of the guides to complex with Cas9 independently. The RNP complexes of both guides were subsequently combined, added to the desired cells as per the manufacturer's instructions, and electroporated. After CRISPR, cells were scaled up and sorted on the MSLN(-) population (R\&D, anti-MSLN pAb or clone \#618923). Bulk-sorted MSLN(-) cells were then screened against MSLN binders in Jurkat cell-based assays.

\section{Selectivity screening using Jurkat cells}

To explore off-target reactivity of MSLN CAR3 Tmod cells, Jurkat cell activation was tested against a diverse panel of target cell lines chosen to encompass the large majority of adult gene expression as described elsewhere (Wang et al, in press). Positive controls were used to confirm that effector cells could be activated by MSLN $(+)$ cell lines, and negative controls to set a baseline for response (see figure 2A, online supplemental tables 1 and 2 for cell line characterization). Briefly, Jurkat-NFAT-Luciferase cells were transiently transfected to express either CARs or CAR3 Tmod. In parallel, endogenous MSLN $(+)$ target cells, along with their respective MSLN(-) control target cells generated using CRISPR as described earlier, were plated at 1e4 target cells in $15 \mu \mathrm{L}$ complete RPMI (containing 10\% FBS and 1\% P/S) per well in a 384-well plate format. Approximately 18 hours posttransfection, 1e4 Jurkat cells were co-cultured with each target cell and activity compared with Gen2 M5 CAR was assessed via luminescence after 6 hours. Luciferase activity was measured using ONE-Step Luciferase Assay System (BPS Bioscience).

\section{Mouse xenograft study}

Blinded in vivo experiments were conducted by Explora BioLabs under Institutional Animal Care and Use Committee (IACUC)-approved protocols. Female NSG (NOD.Cg-Prkdcscid Il2rgtm1Wjl/SzJ) mice 5-6 weeks old were purchased from The Jackson Labs. Animals were acclimated to the housing environment prior to the initiation of the study. Animals (10/cohort) were implanted subcutaneously with 5e6 MS751 firefly luciferase(+) HLA-A KO tumor or renilla luciferase $(+) \mathrm{A}^{* 02}$ transgenic "normal" cells mixed 1:1 with Matrigel in $100 \mu \mathrm{L}$ on their right and left flanks, respectively, in half of the mice in each cohort, and vice versa in the other half to control for flank growth variation. When tumors reached an average of $100-150 \mathrm{~mm}^{3}$ each $(\mathrm{V}=\mathrm{L} \times \mathrm{W} \times \mathrm{W} / 2)$, animals were randomized into five groups $(n=10$ mice per group) and 2e7 T cells, or saline control, were administered via the tail vein. Prior to injection, $\mathrm{T}$ cells were $\sim 60 \% \mathrm{CAR}(+)$ or $\operatorname{Tmod}(+)$. Post $\mathrm{T}$ cell injection, graft measurements by calipers were performed $3 \times$ times per week and BLI $1 \times$ time per week for the duration of the study. At each BLI session, Rediject Coelenterazine h substrate (PerkinElmer) was first injected to visualize renilla luciferase $(+)$ "normal" cells, followed by injection of XenoLight D-Luciferin Potassium Salt (PerkinElmer) 6 hours later to visualize firefly luciferase $(+)$ tumor cells on the reverse flank.

Blood was collected 5 and 12 days after $T$ cell injection and at the time of study termination for flow analysis, along with serum. At each time point, human $\mathrm{T}$ cells in the blood were quantified by flow cytometry (BD FACSCanto II) post RBC lysis. Cells were stained with antimouse CD45-FITC (clone 30-F11), anti-human CD3-PE (clone SK7), anti-human CD4-APC (clone OKT4), and anti-human CD8 $\alpha$-PerCP-Cy5.5 (clone RPA-T8). In a separate panel, memory and effector cell phenotype analysis was assessed by staining with anti-human CD62L-PE (clone DREG-56) and anti-human CD45RO-APC (clone UCHL1). All antibodies were obtained from Biolegend and used at a 1:50 dilution. A fixable near IR live/dead stain (Invitrogen) was used to exclude dead cells from analysis and AccuCheck Counting Beads (Invitrogen) were used for reference. Serum cytokine analysis was 
A

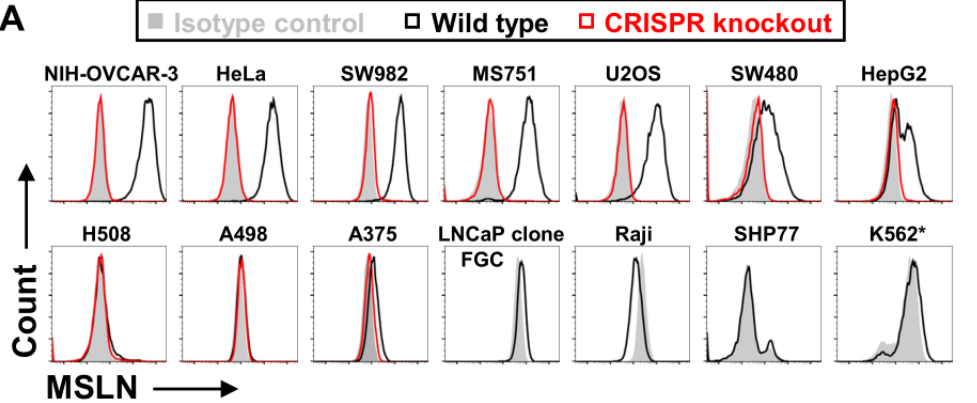

C

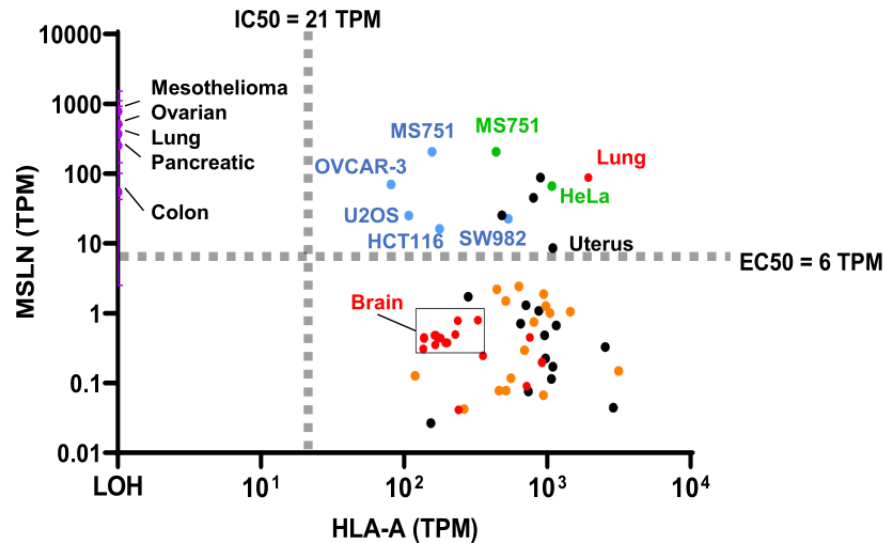

D

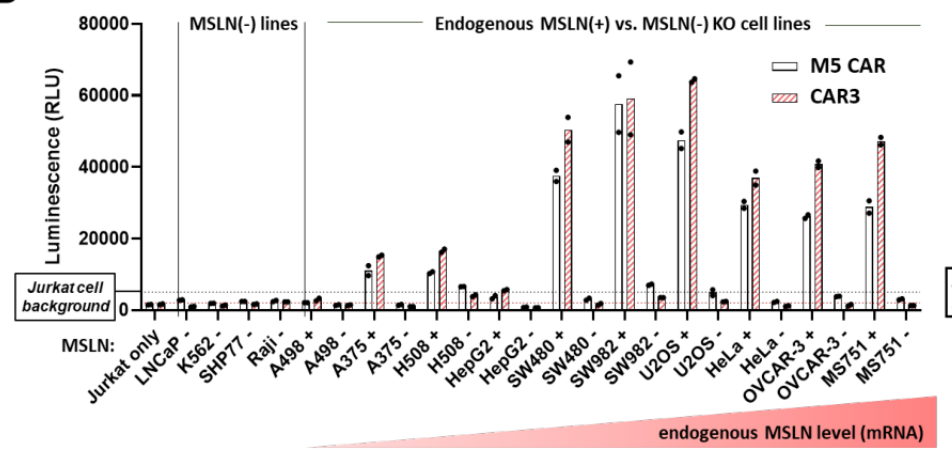

B

MSLN

HLA-A
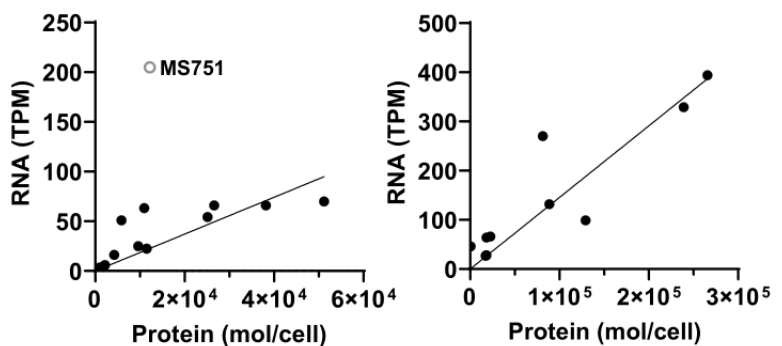

- Vital organs

- Supplementary to vital organs

- Other tissues

- Tumors

- $A^{*} 02$ transgenic cell lines

- Endogenous $A^{*} 02$ cell lines

Figure 2 MSLN Tmod functions selectively with human cell lines \pm MSLN expression. (A) Expression of MSLN in human cell lines assessed by staining with MSLN mAb and flow cytometry. K562 displayed some cross-reactivity to the anti-MSLN antibody, although no functional reactivity to CAR3 or benchmark M5 CAR was observed. (B) Levels of MSLN and $A^{*} 02$ mRNA (CCLE) and protein (QIFIKIT) show correlation. Conversion between protein and mRNA levels was calculated using these standard curves (see online supplemental table 1). (C) $E_{50}$ and IC $\mathrm{C}_{50}$ of construct in relation to MSLN and HLA-A expression levels in normal (GTEx database) and tumor tissues and cell lines (TCGA, CCLE databases). Conversions between protein and mRNA levels were calculated using the standard curves shown in figure 2B. HeLa and MS751 A*02 transgenic cell line variants shown on the graph (green) better mimic activator and blocker target ratios in normal tissues. Only tissues above the $E_{50}$ line express sufficient levels of MSLN to activate CAR3. Tissues above the IC50 line express sufficient levels of $A^{*} 02$ to engage the blocker. Therefore, all normal tissues are expected to be spared by CAR3 Tmod, including the lung. Even those vital tissues in the brain with relatively low HLA-A expression ( 100 TPM) are still well above the $\mathrm{IC}_{50}$ for MSLN Tmod blocker function. In addition, MSLN expression in brain is extremely low ( 1 TPM), well below the $\mathrm{EC}_{50}$ for activation. Organs were classified as vital or supplementary to vital per ICH Guidance S7A (https://www.fda.gov/media/72033/download). (D) CAR3 selectivity benchmarked against Gen2 M5 CAR on a panel of human cell lines. Activation of CARs in a Jurkat cell functional assay by MSLN(+) or MSLN(-) cell lines showed that both CARs display MSLN-specific activation. (E) MSLN CAR3 Tmod construct selectivity in a Jurkat cell functional assay on a subset of MSLN(+) and MSLN(-) control target cells showed no offtarget activity. Note that the blocker slightly reduces background or tonic activation compared with CAR alone. This has been observed generally by us and by others (Hwang et al, 2021). Two-way ANOVA with a multiple comparisons test was used to calculate significance between CAR3 and CAR3 Tmod. Data shown for D-E depict the average of technical replicates. All experiments were run independently at least three times.

conducted using the BD Bioscience human inflammatory cytokine cytometric bead array per manufacturer's instructions.
CRISPR knockout of B2M in primary T cells

Frozen PBMCs were thawed and activated. Transductions were performed 24 hours post-activation using lentivirus 
(Alstem) at MOI 10. 24 hours post-transduction, primary T cells were transfected with CRISPR-Cas9:sgRNA complexes. Briefly, cells were collected and washed with PBS before resuspending in supplemented P3 nucleofection buffer (Lonza). A quantity of 20 pmol of Cas9 (Synthego) was combined with 130 pmol B2M-targeting sgRNA (Synthego) and incubated in P3 nucleofection buffer before addition to cells. Twenty microliters of the cell and ribonucleoprotein (RNP) mixture was transferred to a 16-well Nucleocuvette Strip and electroporated with the $4 \mathrm{D}$ nucleofector using the stimulated $\mathrm{T}$ cell program (EO-115). The cells were recovered in $100 \mu \mathrm{L}$ of prewarmed media, X-VIVO 15 (Lonza) supplemented with $5 \%$ HIA human AB serum and $300 \mathrm{IU} / \mathrm{mL}$ IL-2. PBMCs were cultured and expanded in LymphoONE Tcell Expansion Medium (Takara Bio) supplemented with 1\% HIA human $\mathrm{AB}$ serum and $300 \mathrm{IU} / \mathrm{mL}$ IL-2 for 6 days. Post expansion, positively transduced primary $\mathrm{T}$ cells were enriched using anti-PE microbeads (Miltenyi) according to manufacturer's instructions against Protein L-biotin:streptavidin-PE using LS column. Cytotoxicity of B2M KO primary $\mathrm{T}$ cells was then assessed exactly as described previously.

\section{Statistical analysis}

Statistical analyses were performed using GraphPad Prism software. All Jurkat cell-based in vitro studies (including mRNA titration experiments) are shown as mean \pm SD of technical replicates, while primary $\mathrm{T}$ cell-based in vitro studies are shown as mean $\pm \mathrm{SD}$ of technical triplicates. Where applicable, technical replicates are shown as individual data points, with bars denoting means. All data are representative of a minimum of $n=2$ experimental repeats, unless otherwise noted. Data for in vivo studies are shown as mean \pm SEM. For mRNA titration studies, curves were fit using a fourparameter non-linear regression analysis. $\mathrm{EC}_{50}$ and $\mathrm{IC}_{50}$ values were calculated directly from the curves. Direct comparisons were analyzed using two-way ANOVA, followed by a multiple comparison's test, unless otherwise noted.

\section{RESULTS \\ MSLN Tmod constructs are blocked efficiently by HLA-A*02 antigen in Jurkat and primary $\mathrm{T}$ cells}

To identify an optimal MSLN CAR Tmod construct that discriminates between tumor and "normal" cells, we tested six CARs, in addition to benchmarks used in the clinic ${ }^{162627}$ (see online supplemental figure 1 for details of CAR isolation and testing). One of these, M5, was clinically active but toxic (Tanyi et al, Penn Medicine presentation, 2021; https://clinicaltrials.gov/ct2/show/ NCT03054298). The CARs displayed a range of sensitivities $\left(\mathrm{EC}_{50}\right)$ and maximal responses $\left(\mathrm{E}_{\max }\right)$ (online supplemental figure $1 \mathrm{E}$ ). Previous work suggested that $\mathrm{E}_{\text {max }}$ is related to surface expression of the receptor and $\mathrm{EC}_{50}$ to its binding and signal transduction properties, especially the ligand-binding domain (LBD). ${ }^{28} 29$ We converted the $\mathrm{EC}_{50}$ from relative response measured by luminescence in Jurkat cells to absolute molecules/cell using standard curves and QIFIKIT methodology. ${ }^{24}$ One of the benchmark CARs (m912) displayed low functional sensitivity, with an $\mathrm{EC}_{50}$ estimated at $\sim 80 \mathrm{~K}$ molecules/ cell. The $\mathrm{EC}_{50}$ values for the other benchmarks and novel CARs ranged downwards of $\sim 6 \mathrm{~K}$ molecules/cell. Several novel LBDs assayed as CARs in Jurkat cells had $\mathrm{EC}_{50}$ values below the limit of detection $(<3 \mathrm{~K}$ molecules/cell). The CARs were then paired with an $A^{*} 02$-directed inhibitory receptor or "blocker" previously shown to inhibit functional response in Jurkat and primary T cells. ${ }^{6}$ This blocker comprised an $\mathrm{A}^{*} 02$-binding $\mathrm{scFv}$ fused to the hinge, transmembrane, and intracellular domains of the ITIM-containing LIR-1 protein (figure 1B). All the CARs were blocked in a ligand-dependent manner by the A*02-directed LIR-1 blocker (figure 1C, online supplemental figure $1 \mathrm{~F}$ ). For target cells, we used the $\mathrm{A}^{*} 02(-)$ cervical carcinoma HeLa cell line that, like many other solid-tumor lines, expresses endogenous MSLN. Additional target cells used throughout these studies were characterized for MSLN and A*02 surface expression (figure 2A, online supplemental tables 1 and 2). Because of the combination of high sensitivity and effective functional pairing with the $A^{*} 02$ blocker, we selected CAR3 for further study as our MSLN Tmod lead activator.

We sought to understand the quantitative features of the MSLN CAR3 Tmod construct in relation to target antigen expression levels. Activation and blocking sensitivity of the Tmod construct was examined in detail by mRNA titration experiments in Jurkat cells (figure 1D). To control MSLN levels in HeLa target cells, we first generated a variant line with MSLN inactivated by CRISPR. mRNA titration experiments enabled estimation of $\mathrm{EC}_{50}$ and $\mathrm{IC}_{50}$ for the MSLN CAR3 Tmod construct. A correlation between MSLN mRNA and surface protein levels (figure 2B) allowed us to graph these parameters in the context of the expression levels of antigens on normal and tumor tissues derived from high-quality public databases (figure 2C). We did the same for HLA-A and used this information to plot the HLA-A and MSLN levels of different tissues relative to the $\mathrm{EC}_{50}$ and $\mathrm{IC}_{50}$ of the MSLN CAR3 Tmod construct. The large majority of normal tissues expressed levels of MSLN well below the $\mathrm{EC}_{50}$ of MSLN Tmod (bottom right quadrant). In contrast, certain tissues including lung expressed MSLN at a level above the $\mathrm{EC}_{50}$ of the Tmod construct and, therefore, are considered high risk absent an effective blocker. However, these at-risk tissues also express HLA-A levels above the Tmod $\mathrm{IC}_{50}$, suggesting they will be protected against cytotoxicity by the $\mathrm{A}^{*} 02$-targeted LIR-1 blocker component of Tmod. We also placed on the graph the cell lines (including transgenic A*02 HeLa and MS751, highlighted in green) that we used to model "normal" and tumor tissues in functional assays.

To systematically explore off-target reactivity of MSLN CAR3 Tmod cells, we tested Jurkat cell activation by a panel of target cell lines chosen to encompass the large majority of adult gene expression (Wang et al, in press; see Methods). We used positive controls to confirm that effector cells could be activated by MSLN $(+)$ cell lines, 
A

HeLa

WT tumor vs. $A^{*} 02(+)$ "normal" $B: A=27$

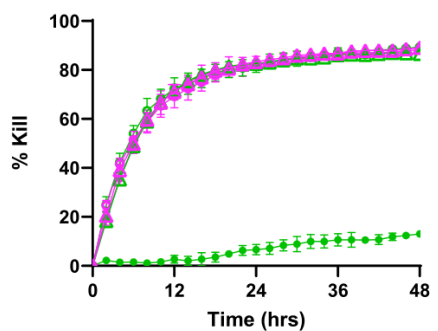

SW982

HLA-A KO tumor vs. WT "normal" $B: A=20$

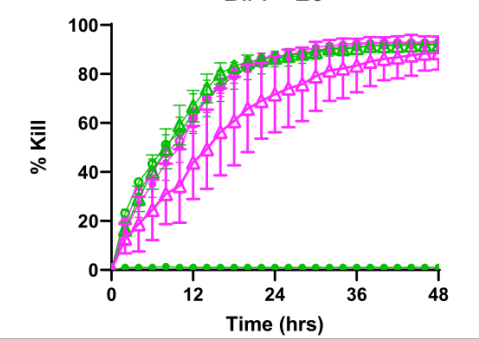

U2OS

HLA-A KO tumor vs. WT "normal"

$B: A=2$

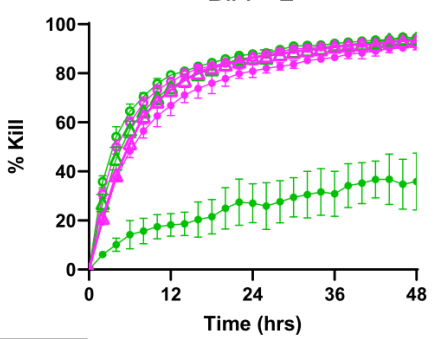

$\triangle$ M5 CAR O CAR3 O CAR3 Tmod

B

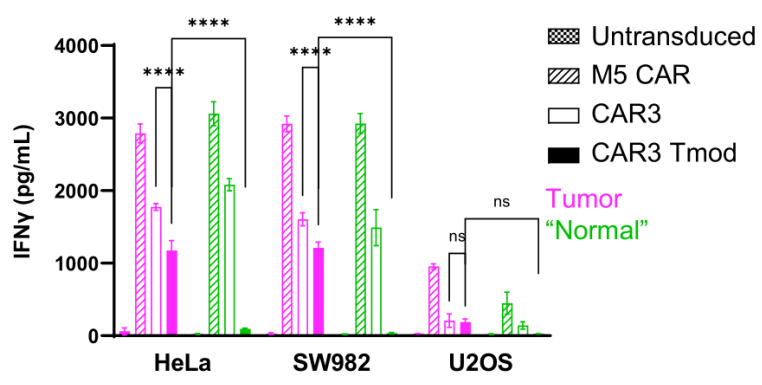

D

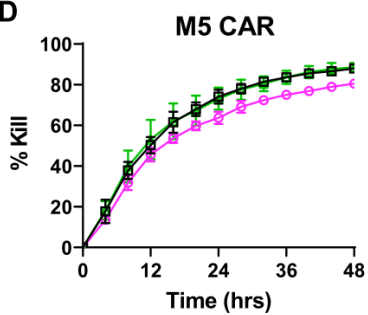

C

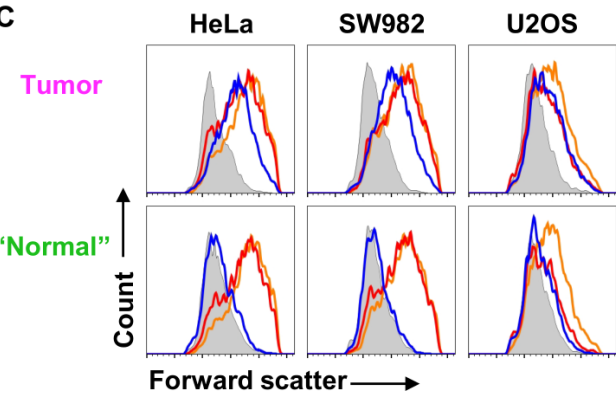

Untransduced, M5 CAR, CAR3, CAR3 Tmod

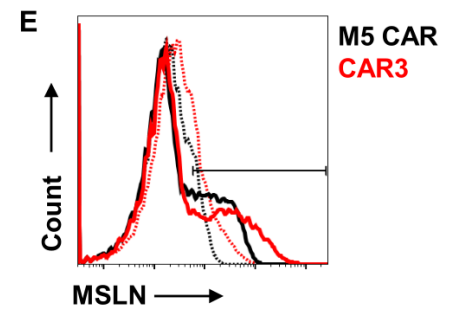

Figure 3 MSLN CAR3 Tmod construct is potent and selective in primary T cell assays. (A) MSLN CARs and CAR3 Tmod cytotoxicity in primary T cells with various MSLN(+)A*02(-)RFP(+) tumor or MSLN(+)A*02(+)GFP(+) "normal" target cells. $A^{*} 02: M S L N(B: A)$ target antigen ratios for each cell line are noted. M5 CAR is Gen2. E:T=1:1. Data are representative of multiple $(3+)$ independent repeats with at least 4 different donors. (B) Secreted IFN- $\gamma$ and (C) T cell activation assessed by forwardscatter shift was measured 48 hours after co-culture of transduced T cells with tumor or "normal" target cells (same as in figure 3A). Two-way ANOVA with a multiple comparisons test was used to calculate significance ( $n=3$ technical replicates). (D) sMSLN does not affect CAR-T activity. Acute cytotoxicity of tumor or "normal" target cells by M5 benchmark CAR or CAR3 was not affected by the presence of $500 \mathrm{ng} / \mathrm{mL}$ sMSLN (Acro Bio). E:T=0.6:1. (E) Staining of transiently transfected CAR(+) Jurkat cells with labeled SMSLN monomer (dashed) or tetramer (solid) analyzed by flow cytometry shows that the sMSLN is structurally intact and able to bind.

and negative controls to set a baseline for response. Both CAR3 and the benchmark M5 CAR displayed MSLNspecific activation and were inactive against MSLN(-) lines (figure 2D). In addition, no detectable responses were triggered in CAR3 Tmod Jurkat cells (figure 2E), despite high sensitivity of the assay, estimated at $\sim<1000$ MSLN molecules/cell.

The MSLN CAR3 Tmod construct was then tested in primary $\mathrm{T}$ cells to confirm its behavior in the intended therapeutic effectors. MSLN benchmark CARs SS1 and M5 were used for comparison (online supplemental figure 2A). Target cells were HeLa cells and variants: (1) native MSLN(+)A*02(-) HeLa cells modeled tumor cells without blocker antigen (magenta); (2) transgenic $A^{*} 02(+)$ variant HeLa cells modeled "normal" mesothelial cells (green).
Combinations of different constructs and target cells demonstrated that all MSLN CAR Tmod constructs killed tumor cells potently and blocked killing in an $\mathrm{A}^{*} 02$-liganddependent fashion (figure 3A). Cytotoxicity was mirrored by IFN- $\gamma$ release (figure $3 \mathrm{~B}$ ) and $\mathrm{T}$ cell activation (figure $3 \mathrm{C}$ ). We then tested if soluble MSLN (sMSLN), which is present in the blood of some patients with cancer at high levels (median $200 \mathrm{ng} / \mathrm{mL}$; range $20-2000 \mathrm{ng} / \mathrm{mL}$ ), ${ }^{30}$ interfered with MSLN CAR3 Tmod cells. sMSLN added to the cultures at $500 \mathrm{ng} / \mathrm{mL}$ had no effect on function of either the CAR or Tmod T cells (figure 3D,E). Thus, the Tmod constructs composed of an A*02-directed blocker and MSLN CAR3 activator displayed high potency and specificity for MSLN(+)A*02(-) target cells in both Jurkat and primary $\mathrm{T}$ cell assays and was unaffected by sMSLN. 
A

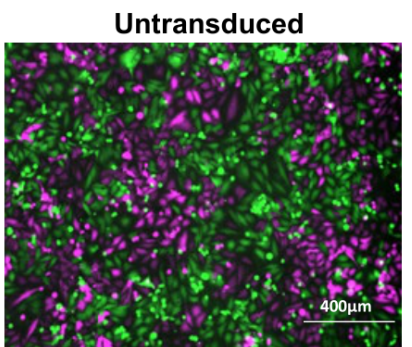

B

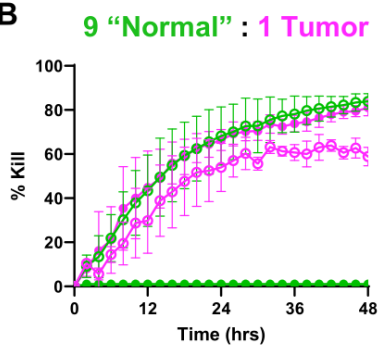

O CAR3

CAR3 Tmod
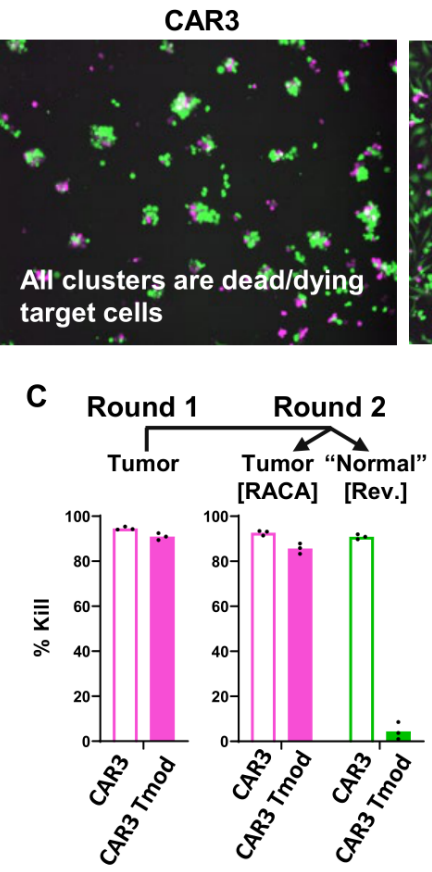

CAR3 Tmod
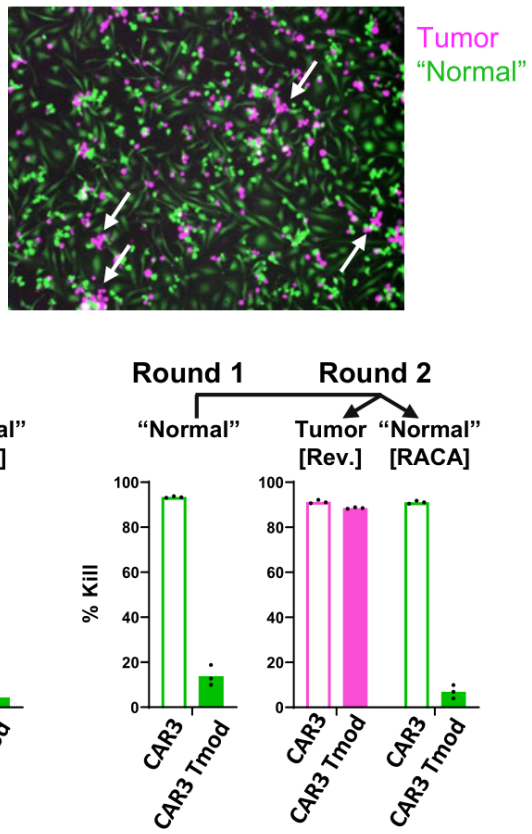

D
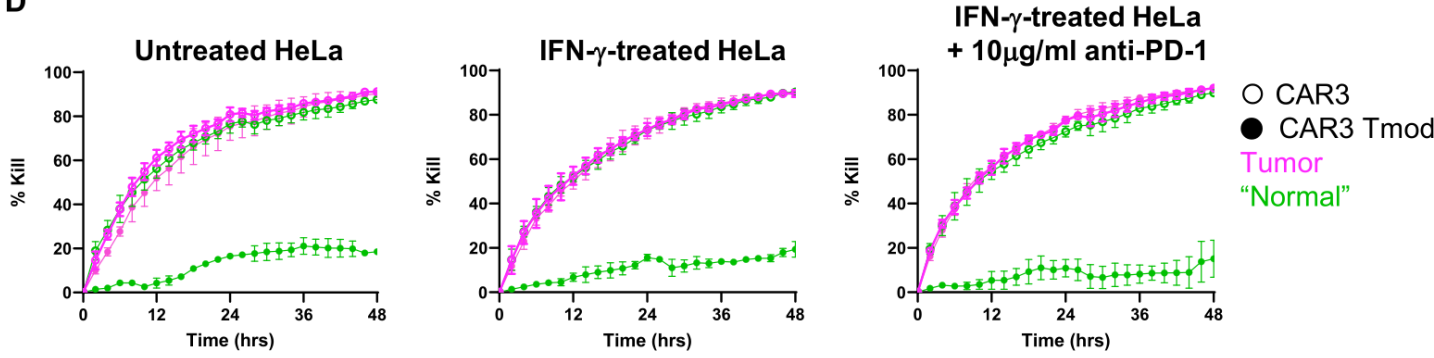

Figure 4 MSLN CAR3 Tmod construct is selective, serially-cytotoxic, and reversible in primary T cell assays. (A) MSLN CAR3 Tmod cells selectively kill RFP(+) tumor cells and spare GFP(+) "normal" cells in mixed tumor/"normal" cell cultures. Due to the adherent nature of the HeLa cell line, dead/dying target cells tend to remain as clusters on the surface. White arrows point to examples of dead/dying RFP(+) tumor cells. (B) Representative cytotoxicity in mixed "normal" and tumor co-cultures with "normal":tumor=9:1 (see online supplemental figure 2C for other ratios). E:T=0.6:1. (C) MSLN CAR3 Tmod construct mediates selective, serially-cytotoxic, and reversible cytotoxicity. After 48 hours of co-culture with tumor or "normal" HeLa target cells, $T$ cells were co-cultured for a second round with either tumor or "normal" target cells; E:T=1.2:1. RACA, repeat antigen-challenge assay; Rev., reversibility assay. Data shown for $\mathrm{C}$ depicts the average of technical triplicates and is representative of at least 2 independent experimental repeats. (D) MSLN CAR3 Tmod killing of tumor target cells and blocking of "normal" target cells is not affected by the presence of PD-L1 induced by overnight treatment of HeLa target cells with $50 \mathrm{ng} / \mathrm{mL} \mathrm{IFN-} \gamma$ (see online supplemental figure 2E). Blockade with anti-PD-1 antibody similarly has no effect on CAR3 Tmod.

\section{MSLN CAR3 Tmod construct mediates selective, reversible cytotoxicity in mixed-cell and serial cultures}

We next tested a variety of other properties of the MSLN CAR3 Tmod construct important in the context of cancer cell therapy. First, we demonstrated that the construct mediates antigen-selective cytotoxicity in mixed cultures of tumor and "normal" target cells, a critical feature of therapeutic $\mathrm{T}$ cells operating in the heterogeneous milieu of a tumor. Native MSLN $(+) \mathrm{A}^{*} 02(-)$ HeLa cells expressing RFP (tumor) were mixed at different ratios with MSLN $(+)$ A*02(+) cells expressing GFP ("normal"). These co-cultures were exposed to $\mathrm{T}$ cells engineered with different receptor constructs and imaged. Whereas CAR-only constructs killed indiscriminately, the Tmod constructs killed the native HeLa tumor cells only, leaving the A*02(+) "normal" cells unharmed (figure 4A,B). Selectivity was detectable even at "normal":tumor cell ratios of 9:1 (figure 4B, online supplemental figure 2B,C). We next examined the capacity of Tmod cells to switch between ON (active killing) and OFF (blocked) states using cytotoxicity as readout. In these experiments, the transduced $\mathrm{T}$ cells were transferred from one batch of target cells to another (figure 4C, online supplemental figure 2D). MSLN Tmod cells engaged in killing tumor cells were able to quickly switch to the OFF state and refrain from killing the "normal" cells after transfer. The reverse was also true. Tmod cells quickly reactivated and killed tumor cells after being exposed for 2 days to "normal" cells where they were quiescent. Together, these results suggested that MSLN CAR3 Tmod mediates selective, reversible cytotoxicity in mixed cultures of tumor and 
A

MSLN CAR or MSLN Tmod

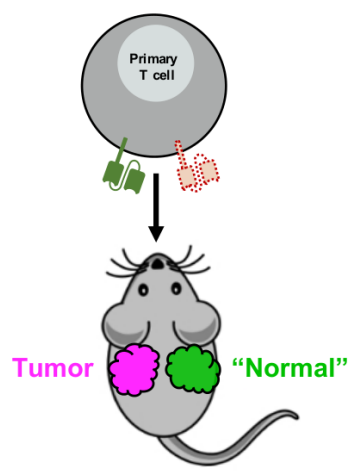

B

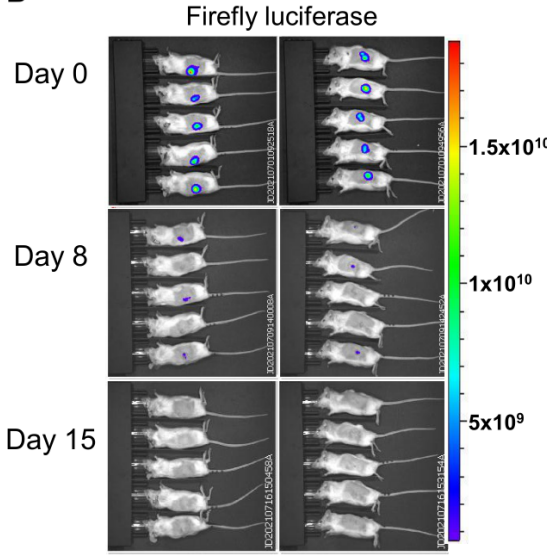

"Normal" Renilla luciferase

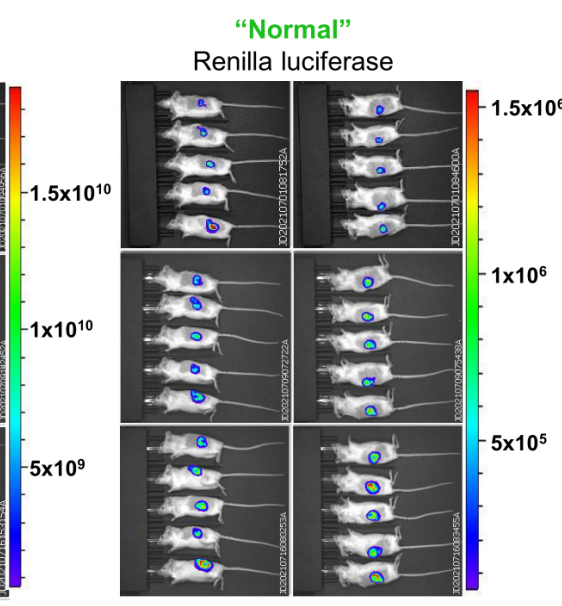

C

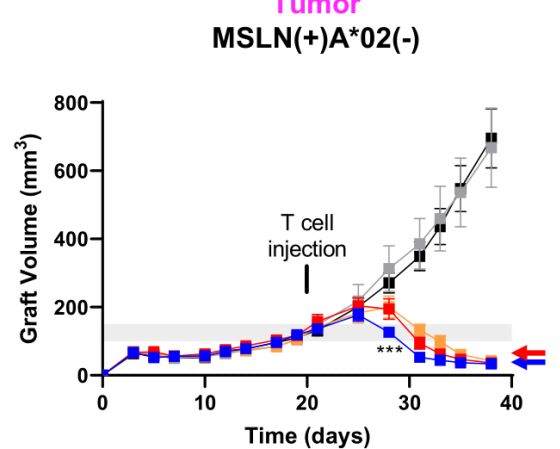

Tumor

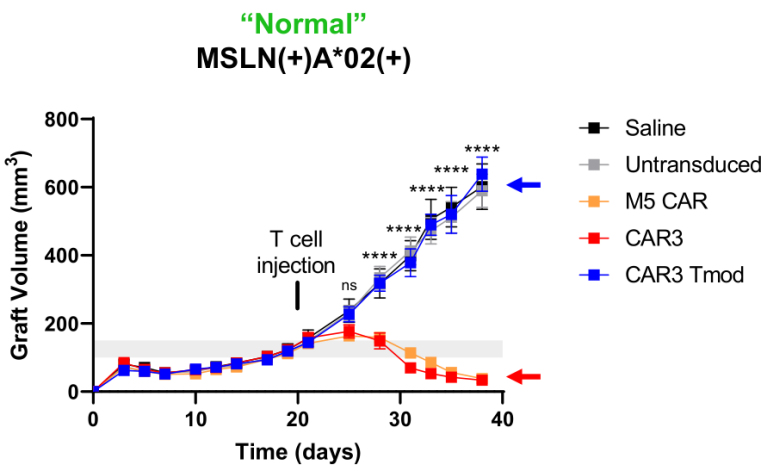

MSLN(+)A*02(+)

Figure 5 Tmod construct mediates selective killing of tumor cells in a xenograft model. (A) Schematic diagram of the dualflank tumor and "normal" MS751 xenograft model. (B) Bioluminescence imaging of "normal" and tumor cells. Bioluminescence values are to the right of the color scale in flux units of photons $/ \mathrm{sec} / \mathrm{cm}^{2} / \mathrm{sr}$. Day $0=$ pre-T cell injection; days 8 and $15=$ post-T cell injection. (C) Graft sizes assessed by caliper measurement. Left: Tmod cells (blue arrow) kill tumor cells equivalent to CARTs (red arrow). Right: CAR-Ts kill, while Tmod cells spare "normal" cells. Two-way ANOVA with a multiple comparisons test was used to calculate significance between CAR3 and CAR3 Tmod at each timepoint. $n=10$ animals per group.

"normal" cells. Finally, CAR3 and CAR3 Tmod cells did not respond differently to upregulation of a second inhibitory stimulus, PD-L1, on target cells by IFN- $\gamma$, suggesting that blocker function is not strongly influenced by checkpoint activation (figure 4D, online supplemental figure 2E). All of these features are consistent with a cell therapy that has the potential to be safe and efficacious in selected patients with cancer and conform to similar effects seen previously in experiments with a CD19 Tmod construct (Hamburger et al, 2020).

\section{MSLN CAR3 Tmod cells selectively kill tumors in a xenograft model}

To examine the behavior of MSLN CAR3 Tmod cells in vivo, we used a mouse xenograft model. HeLa cells did not grow well in the immunocompromised (NSG) mice, so we developed another cervical cancer cell line (MS751) for grafted target cells. Again, we used endogenous MSLN as the activator antigen and engineered the blocker antigen $A * 02$ by gene transfer to better approximate "normal" tissue levels (online supplemental table S1). MSLN(+)A*02(-) MS751 cells, generated by CRISPR knockout of $\mathrm{A}^{*} 02$, modeled tumor (magenta) while the MSLN $(+) \mathrm{A}^{* 02(+)}$ transgenic variant modeled "normal" cells (green) in these in vivo experiments. The cell lines were also engineered with luciferase to enable bioluminescence as an independent readout of tumor survival and growth. Selective killing and blocking by the MSLN CAR3 Tmod construct of engineered MS751 tumor and "normal" target cells was first confirmed in vitro (online supplemental figure 3A). Then mice (10/cohort) were implanted with tumor and "normal" cells on opposite flanks (figure $5 \mathrm{~A}$ ). When xenografts reached $\sim 100-150 \mathrm{~mm}^{3}$ in volume, mice were infused via tail vein with $2 \mathrm{E} 7$ total $\mathrm{T}$ cells/mouse. After a short delay, both the CARs and the Tmod constructs killed the MSLN $(+) \mathrm{A}^{*} 02(-)$ tumor cells equally well measured by bioluminescence intensity and caliper (figure 5B,C, online supplemental figure 3B,C). Whereas the CAR-only T cells killed both tumor and "normal" grafts with equal effectiveness, Tmod cells killed only the tumor grafts. In the presence of Tmod cells, the "normal" cell grafts grew comparably with those in the control groups treated with untransduced $\mathrm{T}$ cells or saline. Interestingly, CAR3 T cell expansion in the peripheral blood was $\sim 10 \times$ higher than observed for CAR3 Tmod cells. Benchmark M5 CAR T cells showed even higher expansion ( $5 \times$ higher than CAR3) 
A

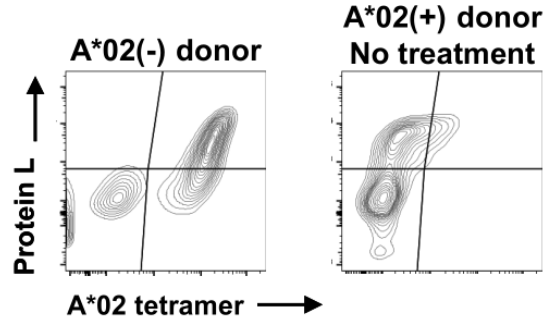

B

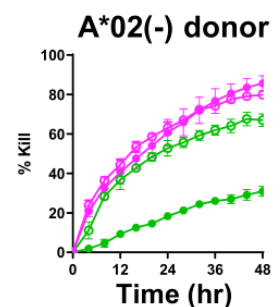

$A^{*}$ 02(+) donor No treatment

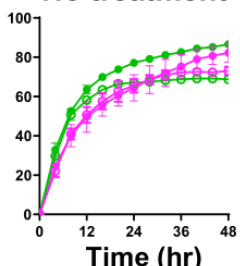

O SS1

SS1 Tmod
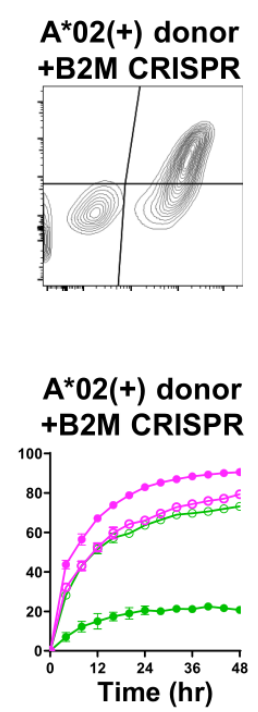
+B2M CRISPR

"Normal"
C
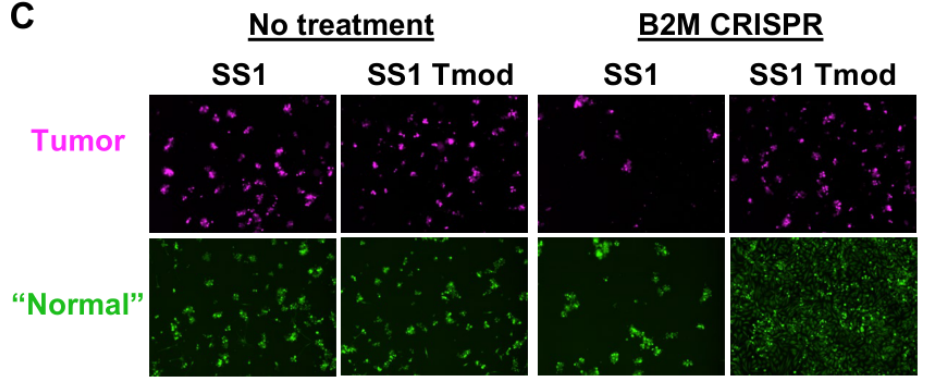

D

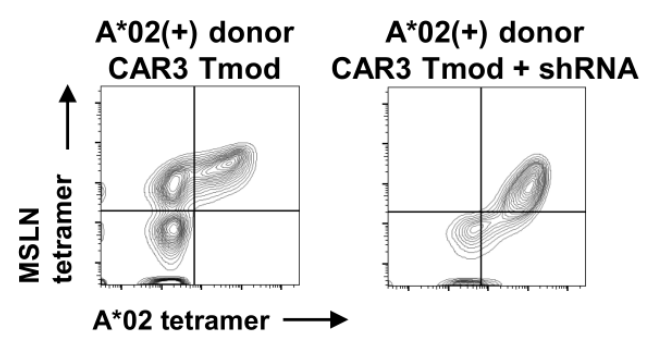

E CAR3 Tmod +/- B2M ShRNA

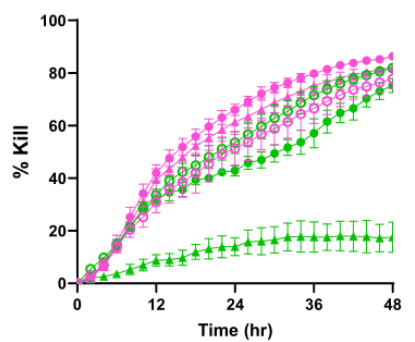

O CAR3

- CAR3 Tmod

- CAR3 Tmod + shRNA

Tumor

"Normal"

$\mathbf{F}$

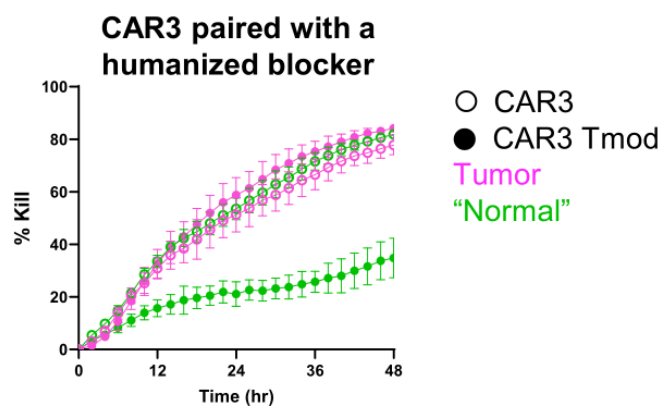

Figure 6 MSLN Tmod system can be extended to autologous T cells. (A) MSLN SS1 CAR Tmod construct kills MSLN(+) $A^{*} 02(-)$ tumor HeLa target cells but no longer blocks in the presence of autologous $A^{*} 02$ as a result of cis-binding. B2M knockout (KO) by CRISPR restores blocker availability as demonstrated by binding to $A^{*} 02$ tetramer. (B) B2M KO by CRISPR in $\mathrm{A}^{*} 02(+) \mathrm{T}$ cell donors restores blocking on MSLN(+)A*02(+) "normal" HeLa target cells similar to $A^{*} 02(-) \mathrm{T}$ cell donors. E:T=1.2:1 (C) Representative co-culture images at 48 hours for figure 6B. (D) Similar to SS1 Tmod, CAR3 Tmod has reduced binding to $A^{*} 02$ tetramer in $A^{*} 02(+) T$ cells. B2M knockdown (KD) with shRNA restores blocker availability. (E) B2M shRNA also restores blocking of cytotoxicity on MSLN(+)A*02(+) "normal" HeLa cells. (F) Interestingly, CAR3 paired with a humanized $A^{*} 02$ blocker retains the ability to block killing of "normal" cells in $A^{\star} 02(+)$ donor T cells, even in the absence of B2M KO or KD.

despite all three groups having equivalent efficacy in vivo (online supplemental figure 3D). This trend correlated with cytokine secretion (online supplemental figure 3E). Both effector and effector memory phenotypes were consistently observed in CAR-T and Tmod cells 12 days post-T cell injection at peak expansion (online supplemental figure 3F). Together, these results dramatically demonstrated selective activity of the MSLN Tmod system in a mammalian body, consistent with other activator/blocker pairs we have tested (Sandberg et al, in press). ${ }^{6}$

\section{MSLN CAR3 Tmod mechanism can function with autologous T cells}

Use of an $\mathrm{A} * 02$-targeted blocker raises the possibility that the blocker may be bound in cis by endogenous $A^{*} 02$ molecules produced in autologous $\mathrm{T}$ cells. Indeed, we observed decreased blocker binding with $\mathrm{A} * 02$ tetramer in transgenic $\mathrm{A}^{*} 02(+)$ Jurkat cells compared with native Jurkat $\mathrm{A}^{*} 02(-)$ cells (online supplemental figure 4). A similar decrease was observed in primary $\mathrm{T}$ cells from
A*02(+) donors transduced with MSLN CAR3 Tmod or SS1 Tmod constructs compared with $\mathrm{A}^{* 02(-)}$ donor $\mathrm{T}$ cells (figure $6 \mathrm{~A}$, online supplemental figure 4 ). More importantly, this decreased binding translated to poor blocker function in both $\mathrm{A}^{* 02(+)}$ Jurkat and primary $\mathrm{T}$ cells relative to their $A^{*} 02(-)$ counterparts (figure $6 \mathrm{~B}, \mathrm{C}$, online supplemental figure 4). These observations illustrated how cis-binding may pose a problem for an autologous MSLN CAR3 Tmod product that uses an $\mathrm{A} * 02$-directed blocker.

To address this problem, we developed solutions based on shRNA or CRISPR inactivation of endogenous $\beta_{2}$ microglobulin (B2M). Because B2M is required for all HLA class I expression, these approaches were expected to mitigate the cis-binding effect of $\mathrm{A}^{*} 02$. As predicted, knockdown or knockout of B2M in primary $\mathrm{T}$ cells restored blocker binding and function to levels comparable with $\mathrm{A}^{*} 02(-)$ donor $\mathrm{T}$ cells (figure $6 \mathrm{~A}-\mathrm{E}$ ). Thus, shRNA- or CRISPR-mediated abrogation of cis-binding 
A

CAR3 A*03 Tmod

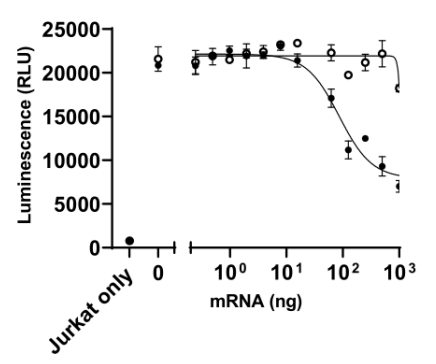

B

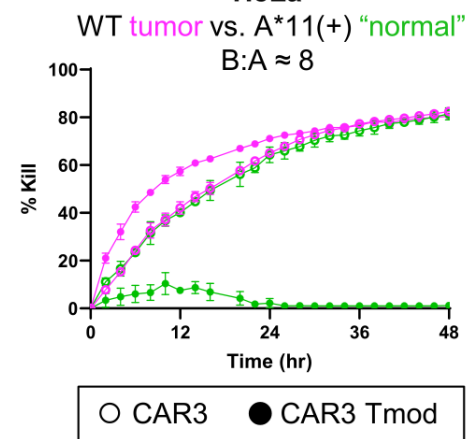

CAR3 B*07 Tmod

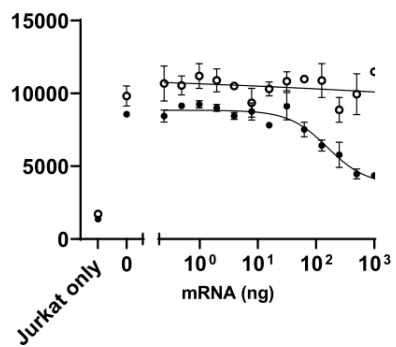

C

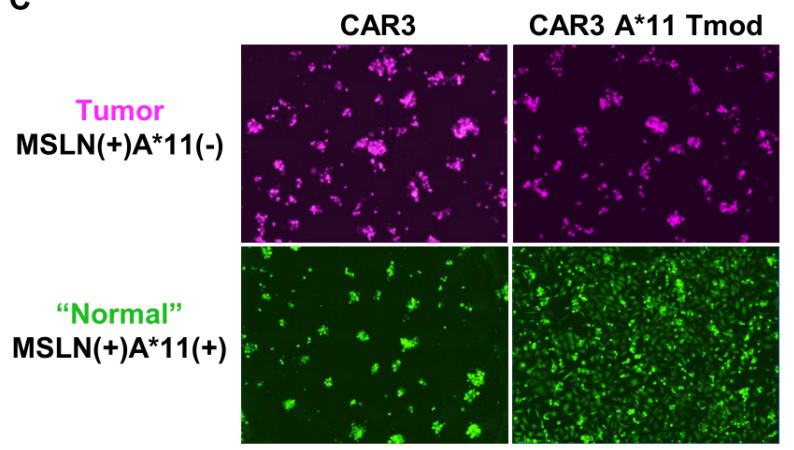

Figure 7 MSLN Tmod system can be extended to other blockers. (A) Jurkat cells expressing MSLN CAR3 and $A^{*} 03, B^{*} 07$ or $A^{*} 11$ blocker constructs were blocked in the presence of increasing blocker antigen on endogenous MSLN(+) HeLa target cells. (B) MSLN CAR3 $A^{*} 11$ Tmod cytotoxicity in primary T cells with MSLN(+)A*11(-)GFP(+) tumor or MSLN(+)A*11(+)GFP(+) "normal" HeLa target cells shows selective blocking in the presence of blocker antigen. Note that both tumor and "normal" target cells used here expressed GFP but are recolored for consistency. $A^{*} 11: M S L N(B: A \approx 8)$ target antigen ratio is noted. E:T=0.8:1. (C) Representative co-culture images at 48 hours for figure 7B.

produced MSLN Tmod cells with functional properties in $\mathrm{A}^{*} 02(+)$ donors that were indistinguishable from function in $\mathrm{A}^{*} 02(-)$ donors. Interestingly, when CAR3 was paired with a blocker containing a humanized scFv, blocker function was mainly retained even in the absence of a cis-binding solution (figure $6 \mathrm{~F}$ ). These results suggest that the MSLN CAR3 Tmod construct may be suitable for allogeneic cell therapy and autologous products.

\section{Tmod mechanism can be extended to other blocker antigens} The impressive performance of the MSLN CAR3 Tmod construct that uses an $\mathrm{A} * 02$-directed blocker suggested the possibility that CAR3 might be paired with blockers gated by other HLA class I alleles. If true, this would potentially allow the Tmod platform to treat patients who are not heterozygous for $A^{*} 02$-a number estimated at $>60 \%$ of the population. ${ }^{31}$ For proof of concept of the modularity of the MSLN Tmod system, we concentrated on A*11, the most common class I allele in Asian populations. ${ }^{31}$ Lacking a good $\mathrm{A}^{*} 11$-specific antibody, we screened for selective scFvs using the same method described for isolation of MSLN scFvs (see online supplemental figure 5 for binder isolation and characterization). One high-performing scFv (A*11-LBD4) was fused to LIR-1 and paired with the MSLN CAR3 activator in Jurkat cells. This $\mathrm{A}^{* 11}$ blocker demonstrated good function with an $\mathrm{IC}_{50}$ estimated at $\sim 37,000$ molecules/cell from mRNA titration experiments (figure 7A). Finally, the MSLN CAR3 paired with the $A^{*} 11$ blocker was shown to function well in primary $\mathrm{T}$ cells with potency and blockade comparable with the MSLN CAR3 Tmod construct that uses A*02 as blocker antigen (figure 7B,C). Encouraged by these results, we converted two other anti-HLA-I monoclonal antibodies, targeting $\mathrm{A}^{*} 03^{21}$ and $\mathrm{B} * 07,{ }^{22}$ to scFvs and tested these constructs as MSLN Tmod blockers (figure 7A). These findings suggest that the Tmod system is sufficiently modular to accommodate pairings of one activator with multiple blockers, implying potential utility of the cell therapy platform for the large majority of patients with HLA LOH.

\section{DISCUSSION}

Given the distribution of MSLN in the adult body, ${ }^{8911}$ it is vital that the blocker functions well with a MSLN-targeted activator. The properties of the $\mathrm{A}^{*} 02$ blocker described here suggest it will inhibit activation signals that arise from the CAR across a broad range of MSLN antigen levels. It is also likely that the blocker will inhibit other sources of $\mathrm{T}$ cell activation triggered by the CAR, including off-target. Because the HLA blocker antigen is ubiquitously expressed in nucleated cells, it should provide an inhibitory signal under all circumstances except when the blocker antigen is absent, as in tumors selected for $\mathrm{LOH}$. The blocker may also prevent cytokine-release syndrome, a continuing source of toxicity observed for $\mathrm{T}$ 
cell therapies, ${ }^{32}{ }^{33}$ if the stimulus involves normal cells or cell fragments.

The MSLN therapeutic candidate described here requires selection of patients who are germline heterozygous for the blocker-antigen allele and whose tumors have lost this allele via LOH. Fortunately, such diagnostic tests have been developed recently which use the enormous power of DNA sequencing technology ${ }^{34-36}$ (Molina et al, 2021 SITC abstract no. 341). DNA-sequence-based $\mathrm{LOH}$ detection has sufficient sensitivity to distinguish most cases of clonal versus subclonal LOH in the tumor, thereby enriching for patients most likely to benefit from the therapy. Because this diagnostic method uses genome sequence from the tumor, it detects any allelic variation and is not restricted to $A * 02$. The lead MSLN Tmod construct described here is applicable to a subset of $\mathrm{A}^{*} 02(+)$ patients. With the goal of extending the Tmod platform to other patients, we have shown that the MSLN CAR3 activator can be paired with blockers directed at three other HLA-I allelic products including $\mathrm{A}^{*} 11 . \mathrm{A} * 11$ is the most frequent HLA-I allele in most Asian populations and represents a significant opportunity for benefit in parts of the world beyond the USA. Indeed, with a collection of blockers directed at six to eight of the most common HLA-I alleles in the world, it should be possible to cover the large majority of solid tumors that harbor LOH at the HLA locus-estimated to be $>15 \%$ of solidtumor mortality at present. ${ }^{27}$ It is also possible that as immunotherapeutics gain broader use, higher frequencies of HLA loss from deletion or expression silencing may arise which may be exploitable by Tmod therapy. In addition, we have shown in a parallel line of work that the $\mathrm{A}^{*} 02$ blocker can be paired effectively with other activators including a CAR directed at another well-known tumor-associated antigen, CEA (Sandberg et al, in press). ${ }^{37}$

The modular Tmod system leverages the capacity of cells-unique among therapeutic modalities-to integrate multiple signals into a coordinated response and thus offers a means to address the fundamental obstacles of solid-tumor therapy ${ }^{5-7}$ Therapeutics must access the tumor and overcome barriers that do not exist for blood cancers where cell therapies have proven effective. ${ }^{38}$ $\mathrm{T}$ cells have an advantage over most other modalities, including antibodies, in that they possess a natural mechanism for active extravasation and biodistribution (see for review Mastrogiovanni $e t a l^{39}$ ). Notably, the CAR3 Tmod cells described here are at least as potent as M5 CAR-T cells that were active but toxic in the clinic, suggesting the Tmod cells will be active in patients at similar doses (Tanyi et al, Penn Medicine presentation, 2021; https://clinicaltrials.gov/ct2/show/NCT03054298). Furthermore, the Tmod approach exploits LOH and mitigates the key constraint on solid-tumor therapeutics posed by normal tissue expression of most targets. Although notoriously non-homogeneous, a large proportion of genetic alterations arise in the founding cell of the neoplasm and are present in all its descendants. ${ }^{40}$ Such homogeneous mutations include LOH and, if such clonal $\mathrm{LOH}$ can be distinguished via a diagnostic test from later alterations present in tumors, LOH heterogeneity at the HLA locus should not constitute a source of resistance. ${ }^{41}$ Unlike single nucleotide substitutions that generate most neoantigens, $\mathrm{LOH}$ is an irreversible event and should be stable even after strong selective pressure is applied via therapy. Finally, as use of checkpoint inhibitors becomes more prevalent, rates of HLA-I loss will likely increase, further expanding the pool of patients who may benefit from MSLN Tmod therapeutics. ${ }^{42}$

Acknowledgements We are grateful to Falene Chai, Christine Yao, James Furey, Dr. Craig Pigott, and Paul Kang for help with MSLN and HLA-A ${ }^{*} 11$ binder screening. We thank William Bretzlaff and Richele Bruno for technical support with functional assays; Casey Gahrs for cloning assistance; Dr. Daniel Nampe, Edwin Liu, and David Ju for assistance with primary T cell preparation for the in vivo study; and Dr. Jun Wang (Explora BioLabs) for overseeing the in vivo study. Melanie Munguia provided key data in figure 6 . We also thank Dr. Armen Mardiros for comments on the manuscript.

Contributors $A K$ is responsible for the overall content as guarantor. AK, AEH, and TT directed the research. TT, GEA, J-YM, BD, SS, DTW, MED, KB, BLL, MSN, and KD designed and conducted experiments.

Funding The authors have not declared a specific grant for this research from any funding agency in the public, commercial or not-for-profit sectors.

Competing interests All authors are current or former employees and shareholders of A2 Biotherapeutics, Inc.

Patient consent for publication Not applicable.

Ethics approval This study does not involve human participants.

Provenance and peer review Not commissioned; externally peer reviewed.

Data availability statement All data relevant to the study are included in the article or uploaded as online supplemental information.

Supplemental material This content has been supplied by the author(s). It has not been vetted by BMJ Publishing Group Limited (BMJ) and may not have been peer-reviewed. Any opinions or recommendations discussed are solely those of the author(s) and are not endorsed by BMJ. BMJ disclaims all liability and responsibility arising from any reliance placed on the content. Where the content includes any translated material, BMJ does not warrant the accuracy and reliability of the translations (including but not limited to local regulations, clinical guidelines, terminology, drug names and drug dosages), and is not responsible for any error and/or omissions arising from translation and adaptation or otherwise.

Open access This is an open access article distributed in accordance with the Creative Commons Attribution Non Commercial (CC BY-NC 4.0) license, which permits others to distribute, remix, adapt, build upon this work non-commercially, and license their derivative works on different terms, provided the original work is properly cited, appropriate credit is given, any changes made indicated, and the use is non-commercial. See http://creativecommons.org/licenses/by-nc/4.0/.

\section{ORCID iD}

Alexander Kamb http://orcid.org/0000-0002-8675-6620

\section{REFERENCES}

1 Dannenfelser R, Allen GM, VanderSluis B, et al. Discriminatory power of combinatorial antigen recognition in cancer $\mathrm{T}$ cell therapies. Cell Syst 2020;11:215-28.

2 Beroukhim R, Mermel CH, Porter D, et al. The landscape of somatic copy-number alteration across human cancers. Nature 2010;463:899-905.

3 Valiante NM, Lienert K, Shilling HG, et al. Killer cell receptors: keeping pace with $\mathrm{MHC}$ class I evolution. Immunol Rev 1997;155:155-64.

4 Cho JH, Okuma A, Sofjan K, et al. Engineering advanced logic and distributed computing in human CAR immune cells. Nat Commun 2021;12:792.

5 Fedorov VD, Themeli M, Sadelain M. PD-1- and CTLA-4-based inhibitory chimeric antigen receptors (iCARs) divert off-target immunotherapy responses. Sci Transl Med 2013;5:215ra172. 
6 Hamburger AE, DiAndreth B, Cui J, et al. Engineered T cells directed at tumors with defined allelic loss. Mol Immunol 2020;128:298-310.

7 Hwang MS, Mog BJ, Douglass J, et al. Targeting loss of heterozygosity for cancer-specific immunotherapy. Proc Natl Acad Sci U S A 2021;118:e2022410118.

8 Chang K, Pai LH, Batra JK. Characterization of the antigen (CAK1) recognized by monoclonal antibody $\mathrm{K} 1$ present on ovarian cancers and normal mesothelium. Cancer Res 1992;52:181-6.

9 Hassan R, Thomas A, Alewine C, et al. Mesothelin immunotherapy for cancer: ready for prime time? JCO 2016;34:4171-9.

10 Bera TK, Pastan I. Mesothelin is not required for normal mouse development or reproduction. Mol Cell Biol 2000;20:2902-6.

11 O'Hara MH, Stashwick C, Plesa G, et al. Overcoming barriers of car T-cell therapy in patients with mesothelin-expressing cancers. Immunotherapy 2017;9:767-80.

12 Chowdhury PS, Viner JL, Beers R, et al. Isolation of a high-affinity stable single-chain Fv specific for mesothelin from DNA-immunized mice by phage display and construction of a recombinant immunotoxin with anti-tumor activity. Proc Natl Acad Sci U S A 1998:95:669-74.

13 Hassan R, Bullock S, Premkumar A, et al. Phase I study of SS1P, a recombinant anti-mesothelin immunotoxin given as a bolus i.v. infusion to patients with mesothelin-expressing mesothelioma, ovarian, and pancreatic cancers. Clin Cancer Res 2007;13:5144-9.

14 Golfier S, Kopitz C, Kahnert A, et al. Anetumab ravtansine: a novel mesothelin-targeting antibody-drug conjugate cures tumors with heterogeneous target expression favored by bystander effect. Mol Cancer Ther 2014:13:1537-48.

15 Luke JJ, Barlesi F, Chung K, et al. Phase I study of ABBV-428, a mesothelin-CD40 bispecific, in patients with advanced solid tumors. $J$ Immunother Cancer 2021;9:e002015.

16 Beatty GL, O'Hara MH, Lacey SF, et al. Activity of mesothelinspecific chimeric antigen receptor T cells against pancreatic carcinoma metastases in a phase 1 trial. Gastroenterology 2018;155:29-32.

17 Ding J, Horton H, Shah S. Abstract 2307: preclinical evaluation of TC-210, a mesothelin-specific T cell receptor (TCR) fusion construct (TRuC ${ }^{\mathrm{TM}}$ ) T cells for the treatment of solid tumors. Cancer Res 2019;79:2307-07.

18 Castelletti L, Yeo D, van Zandwijk N, et al. Anti-Mesothelin CAR T cell therapy for malignant mesothelioma. Biomark Res 2021:9:11.

19 Adusumilli PS, Zauderer MG, Rusch VW, et al. Regional delivery of mesothelin-targeted CAR T cells for pleural cancers: safety and preliminary efficacy in combination with anti-PD-1 agent. J Clin Oncol 2019;37:2511-11.

20 Parham P, Bodmer WF. Monoclonal antibody to a human histocompatibility alloantigen, HLA-A2. Nature 1978;276:397-9.

21 Berger ANNE, Davis JE, Cresswell P. Monoclonal antibody to HLA-A3. Hybridoma 1982;1:87-90.

22 Brodsky FM, Parham P, Barnstable CJ, et al. Monoclonal antibodies for analysis of the HLA system. Immunol Rev 1979;47:3-61.

23 Wang X, Sandberg ML, Martin AD. Potent, selective CARs as potential T-cell therapeutics for HPV-positive cancers. J Immunother 2021.

24 Smith KB, Ellis SA. Standardisation of a procedure for quantifying surface antigens by indirect immunofluorescence. J Immunol Methods 1999;228:29-36.
25 Böhmer RM, Bandala-Sanchez E, Harrison LC. Forward light scatter is a simple measure of T-cell activation and proliferation but is not universally suited for doublet discrimination. Cytometry 2011;79A:646-52.

26 Feng $\mathrm{Y}, \mathrm{Xiao} \mathrm{X}, \mathrm{Zhu} Z$, et al. A novel human monoclonal antibody that binds with high affinity to mesothelin-expressing cells and kills them by antibody-dependent cell-mediated cytotoxicity. Mol Cancer Ther 2009;8:1113-8

27 Klampatsa A, Dimou V, Albelda SM. Mesothelin-targeted CAR-T cell therapy for solid tumors. Expert Opin Biol Ther 2021:21:473-86.

28 James SE, Greenberg PD, Jensen MC, et al. Mathematical modeling of chimeric TCR triggering predicts the magnitude of target lysis and its impairment by TCR downmodulation. J Immunol 2010;184:4284-94.

$29 \mathrm{Xu} \mathrm{H}$, Hamburger AE, Mock J-Y, et al. Structure-function relationships of chimeric antigen receptors in acute $T$ cell responses to antigen. Mol Immunol 2020;126:56-64.

30 Hassan R, Sharon E, Thomas A, et al. Phase 1 study of the antimesothelin immunotoxin SS1P in combination with pemetrexed and cisplatin for front-line therapy of pleural mesothelioma and correlation of tumor response with serum mesothelin, megakaryocyte potentiating factor, and cancer antigen. Cancer 2014;120:3311-9.

31 Gragert L, Madbouly A, Freeman J, et al. Six-locus high resolution HLA haplotype frequencies derived from mixed-resolution DNA typing for the entire US donor registry. Hum Immunol 2013;74:1313-20.

32 Shimabukuro-Vornhagen A, Gödel P, Subklewe M, et al. Cytokine release syndrome. J Immunother Cancer 2018;6:56.

33 Tanyi JL, Stashwick C, Plesa G, et al. Possible compartmental cytokine release syndrome in a patient with recurrent ovarian cancer after treatment with mesothelin-targeted CAR-T cells. J Immunother 2017; 40:104-7.

34 Beaubier N, Tell R, Lau D, et al. Clinical validation of the tempus xT next-generation targeted oncology sequencing assay. Oncotarget 2019;10:2384-96.

35 The ICGC/TCGA Pan-Cancer Analysis of Whole Genomes Consortium. Pan-cancer analysis of whole genomes. Nature 2020;578:82-93

36 Ford L, Wolford JE, Brown SM, et al. A profile on the FoundationFocus CDxBRCA tests. Expert Rev Mol Diagn 2020;20:285-92.

37 Gold P, Freedman SO. Specific carcinoembryonic antigens of the human digestive system. J Exp Med 1965;122:467-81.

38 Roex G, Feys T, Beguin Y, et al. Chimeric antigen receptor-T-cell therapy for B-cell hematological malignancies: an update of the pivotal clinical trial data. Pharmaceutics2020;12:194

39 Mastrogiovanni M, Juzans M, Alcover A, et al. Coordinating cytoskeleton and molecular traffic in T cell migration, activation, and effector functions. Front Cell Dev Biol 2020;8:591348.

40 López S, Lim EL, Horswell S, et al. Interplay between whole-genome doubling and the accumulation of deleterious alterations in cancer evolution. Nat Genet 2020;52:283-93.

41 Foo J, Michor F. Evolution of acquired resistance to anti-cancer therapy. J Theor Biol 2014;355:10-20.

42 Montesion M, Murugesan K, Jin DX, et al. Somatic HLA class I loss is a widespread mechanism of immune evasion which refines the use of tumor mutational burden as a biomarker of checkpoint inhibitor response. Cancer Discov 2021;11:282-92. 